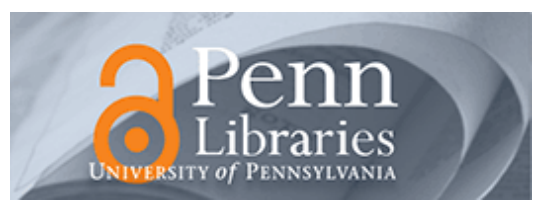

University of Pennsylvania

ScholarlyCommons

10-18-2007

\title{
Computation of D-brane instanton induced superpotential couplings: Majorana masses from string theory
}

\author{
Mirjam Cvetič \\ University of Pennsylvania, cvetic@cvetic.hep.upenn.edu \\ Robert Richter \\ University of Pennsylvania \\ Timo Weigand \\ University of Pennsylvania
}

Follow this and additional works at: https://repository.upenn.edu/physics_papers

Part of the Physics Commons

\section{Recommended Citation}

Cvetič, M., Richter, R., \& Weigand, T. (2007). Computation of D-brane instanton induced superpotential couplings: Majorana masses from string theory. Retrieved from https://repository.upenn.edu/ physics_papers/122

Suggested Citation:

M. Cvetič, R. Richter and T. Weigand. (2007). "Computation of D-brane instanton induced superpotential couplings: Majorana masses from string theory." Physical Review D. 76, 086002.

(c) 2007 The American Physical Society.

http://dx.doi.org/10.1103/PhysRevD.76.086002

This paper is posted at ScholarlyCommons. https://repository.upenn.edu/physics_papers/122

For more information, please contact repository@pobox.upenn.edu. 


\title{
Computation of D-brane instanton induced superpotential couplings: Majorana masses from string theory
}

\author{
Abstract \\ We perform a detailed conformal field theory analysis of D2-brane instanton effects in four-dimensional \\ type IIA string vacua with intersecting $D 6$-branes. In particular, we explicitly compute instanton induced \\ fermion two-point couplings which play the role of perturbatively forbidden Majorana mass terms for \\ right-handed neutrinos or MSSM $\mu$ terms. These results can readily be extended to higherdimensional \\ operators. In concrete realizations of such nonperturbative effects, the Euclidean D2-branehas to wrap a \\ rigid, supersymmetric cycle with strong constraints on the zero-mode structure. Their implications for \\ type IIA compactifications on the $T^{6} /\left(Z_{2} \times Z_{2}\right)$ orientifold with discrete torsion are analyzed. We also \\ construct a local supersymmetric GUT-like model allowing for a class of Euclidean D2-branes whose \\ fermionic zero modes meet all the constraints for generating Majorana masses in the \\ phenomenologically allowed regime. Together with perturbatively realized Dirac masses, these \\ nonperturbative couplings give rise to the seesaw mechanism.

\section{Disciplines} \\ Physical Sciences and Mathematics | Physics

\section{Comments} \\ Suggested Citation: \\ M. Cvetič, R. Richter and T. Weigand. (2007). "Computation of D-brane instanton induced superpotential \\ couplings: Majorana masses from string theory." Physical Review D. 76, 086002. \\ (C) 2007 The American Physical Society. \\ http://dx.doi.org/10.1103/PhysRevD.76.086002
}




\title{
Computation of D-brane instanton induced superpotential couplings: Majorana masses from string theory
}

\author{
Mirjam Cvetič, ${ }^{*}$ Robert Richter, ${ }^{\dagger}$ and Timo Weigand ${ }^{\ddagger}$ \\ Department of Physics and Astronomy, University of Pennsylvania, Philadelphia, Pennsylvania 19104-6396, USA
}

(Received 16 May 2007; published 18 October 2007)

\begin{abstract}
We perform a detailed conformal field theory analysis of D2-brane instanton effects in fourdimensional type IIA string vacua with intersecting D6-branes. In particular, we explicitly compute instanton induced fermion two-point couplings which play the role of perturbatively forbidden Majorana mass terms for right-handed neutrinos or MSSM $\mu$ terms. These results can readily be extended to higherdimensional operators. In concrete realizations of such nonperturbative effects, the Euclidean D2-brane has to wrap a rigid, supersymmetric cycle with strong constraints on the zero-mode structure. Their implications for type IIA compactifications on the $T^{6} /\left(\mathbb{Z}_{2} \times \mathbb{Z}_{2}\right)$ orientifold with discrete torsion are analyzed. We also construct a local supersymmetric GUT-like model allowing for a class of Euclidean D2-branes whose fermionic zero modes meet all the constraints for generating Majorana masses in the phenomenologically allowed regime. Together with perturbatively realized Dirac masses, these nonperturbative couplings give rise to the seesaw mechanism.
\end{abstract}

DOI: 10.1103/PhysRevD.76.086002

PACS numbers: $11.25 . \mathrm{Mj}$

\section{INTRODUCTION}

D-brane instantons in four-dimensional $\mathcal{N}=1$ supersymmetric string compactifications have the potential to generate perturbatively absent matter couplings of considerable phenomenological importance [1-4]. This mechanism relies on the existence of perturbative global Abelian symmetries of the effective action which are the remnants of $U(1)$ gauge symmetries broken by a Stückelberg-type coupling. Under suitable conditions, nonperturbative effects can break these global symmetries by inducing $U(1)$ charge violating effective couplings which are forbidden perturbatively. Because of its phenomenological relevance, the main focus of $[1,3]$ has been on the generation of a large Majorana mass term for right-handed neutrinos. It has been shown that in principle, the peculiar intermediate scale in the range $\left(10^{8}-10^{15}\right) \mathrm{GeV}$ of this term can arise quite naturally and without drastic fine-tuning from the genuinely stringy nonperturbative physics. Finding a concrete realization of this scenario would therefore constitute an interesting example of how string theory provides a natural explanation for an otherwise poorly understood origin of mass scales in particle physics. Other important coupling terms potentially generated in a similar manner include the hierarchically small $\mu$ term of the MSSM $[1,3,5]$ or the Affleck-Dine-Seiberg superpotential of SQCD $[4,6]$. Similar D-brane instanton effects may also give rise to a realistic pattern of Yukawa couplings [7] and play an important role in the context of flux compactifications and de Sitter uplifting [2].

While both the presence of the mentioned perturbative Abelian symmetries and their nonperturbative breakdown

\footnotetext{
*cvetic@cvetic.hep.upenn.edu

rrichter@sas.upenn.edu

†imo@sas.upenn.edu
}

are typical of many different types of string constructions, they are particularly obvious in the context of fourdimensional D-brane models. ${ }^{1}$ For definiteness let us focus on type IIA orientifolds involving intersecting D-branes (see, e.g., [10-12] for up-to-date background material and references). Dual constructions on the mirror symmetric type IIB and type I side have been studied in [4] (see also [5]) and [13], respectively. In our case, the relevant nonperturbative objects are E2-instantons, i.e., Euclidean $D 2$-branes wrapping supersymmetric three-cycles of the internal compactification manifold. These can induce superpotential couplings of the form

$$
W_{n p}=\prod_{i} \Phi_{i} e^{-\left(2 \pi / \ell_{s}^{3} g_{s}\right) \operatorname{Vol}_{E 2}}
$$

between the chiral charged matter superfields.

At the intersection of the E2-instanton wrapping the sLag $\Xi$ and a stack of $N_{a} D 6$-branes on, say, cycle $\Pi_{a}$, there exist $\left[\Xi \cap \Pi_{a}\right]^{+}$and $\left[\Xi \cap \Pi_{a}\right]^{-}$fermionic zero modes $\lambda_{a}$ and $\bar{\lambda}_{a}$ in the fundamental and antifundamental representation of $U\left(N_{a}\right)$, respectively. It follows that the charge of the E2-instanton under the corresponding global $U(1)_{a}$ symmetry is determined by the topological intersection of the instanton cycle $\Xi$ with $\Pi_{a}$ and its orientifold image $\Pi_{a}^{\prime}$ as [1]

$$
Q_{a}=N_{a} \Xi \circ\left(\Pi_{a}-\Pi_{a}^{\prime}\right) .
$$

As a result, the exponential suppression factor in (1) can carry just the right global $U(1)$ charge to cancel the charge of the operator $\prod_{i} \Phi_{i}$, thus rendering the complete term (1) invariant.

\footnotetext{
${ }^{1}$ Similar effects also arise in the S-dual heterotic picture for compactifications with $U(n)$ bundles $[8,9]$.
} 
A detailed prescription for the conformal field theory (CFT) computation of the so induced interaction terms has been given in [1]. ${ }^{2}$ Important constraints on the instanton cycle $\Xi$ beyond the necessary condition of $U(1)$ charge cancellation arise from the requirement of absence of additional uncharged instanton zero modes apart from the usual four bosonic modes $x_{E}^{\mu}$ and their fermionic partners $\theta^{\alpha}$, $\alpha=1$, 2. In particular, the cycle $\Xi$ has to be rigid so that there exist no reparametrization zero modes and it has to satisfy $\Xi \cap \Xi^{\prime}=0$ to avoid zero modes at the intersection of $\Xi$ with its orientifold image. The presence of additional zero modes is expected to give rise to higher fermion couplings as opposed to contributions to the superpotential of the form (1) (see [16] for a discussion of such terms arising from nonisolated world sheet instantons in heterotic $(0,2)$ models). The zero-mode constraints are the main obstacle to the construction of concrete string vacua featuring the described nonperturbative couplings, and in fact no example of an E2-instanton satisfying all of them has been given in the literature so far. For an illustration of the associated challenges in semirealistic toroidal model building see [3]. Besides determining if these constraints can be realized at all, it is also important to study if the scale of the induced couplings can indeed account for the peculiar hierarchies associated to them in the MSSM.

The aim of this article is to provide evidence that these questions can be answered in the affirmative. We do so by constructing a local setup of a toroidal intersecting brane world and explicitly compute the E2-instanton induced Majorana mass terms.

For this purpose, we first need to continue and extend the study of the CFT computation of E2-instanton effects initiated in [1]. This includes, among other things, a careful construction of the vertex operators for the twisted zero modes between the instanton and the D6-branes in Sec. II A. We then determine in Sec. IIC the basic treelevel four-point amplitudes which are the building blocks for nonperturbative couplings of the type (1). Special care requires the analysis of the family replication structure. We exemplify this point in Sec. II D.

In Sec. III, we apply our results to a concrete, but local model meeting all criteria for the generation of $E 2$-induced Majorana mass terms. While, unlike on general Calabi-Yau manifolds, the underlying CFT of toroidal models is exactly solvable and a large class of supersymmetric threecycles is known explicitly, it is much harder to construct rigid cycles in this framework as required for the instanton sector. The only known examples of rigid special Lagrangians on toroidal backgrounds have been given in [17] for the $\mathbb{Z}_{2} \times \mathbb{Z}_{2}$ orientifold with torsion (called $\mathbb{Z}_{2} \times$ $\mathbb{Z}_{2}^{\prime}$ in the sequel) and, more generally, in [18] for shift

\footnotetext{
${ }^{2}$ For previous studies of the CFT associated with the D3$D(-1)$ system see $[14,15]$. Note that $E 2$-instantons at general angles differ from this construction in that they do not correspond to gauge instantons.
}

orientifolds. For the dual constructions with magnetized branes see $[19,20]$.

We construct a local supersymmetric $S U(5)$ GUT-like toy model on the $\mathbb{Z}_{2} \times \mathbb{Z}_{2}^{\prime}$ orientifold with right-handed neutrinos. While its spectrum is far from realistic and fails to satisfy all global consistency conditions, the purpose of this setup is to demonstrate that appropriate instanton sectors contributing to the superpotential of the form (1) do exist in the geometric regime as proposed in [1,3]. In fact, this is the first example even of a local model with these properties. We classify the set of supersymmetric E2-instantons on factorizable cycles whose zero-mode structure allows for superpotential two-fermion couplings of the above type. There are 64 such rigid cycles lying on top of one of the orientifold planes and differing by their twisted charges. Summing up their contributions gives rise to Majorana masses for the right-handed neutrinos of the order of $10^{11} \mathrm{GeV}$.

\section{D2-BRANE INSTANTONS IN TYPE IIA ORIENTIFOLDS}

In this section we summarize and provide additional details on the computation of instanton corrections from Euclidean D2-branes (E2-branes). While the general framework has been described in [1] (see also [3]), Sec. II A clarifies the derivation of the instanton zero modes in some detail and contains a careful construction of the associated vertex operators in toroidal orientifolds. Section IIC describes the exact CFT computation of a prototype of instanton induced couplings, followed by an illustrative example in Sec. II D

\section{A. (Anti-)instantons, zero modes, and vertex operators}

Consider a type IIA orientifold with spacetime filling D6-branes in the presence of a single Euclidean D2-brane wrapping the three-cycle $\Xi$ of the internal manifold. In general, if $\Xi$ is not invariant under the orientifold action we have to consider also the image $D 2$-brane wrapping $\Xi^{\prime}$.

For this topological sector to correspond to a local minimum of the full string action, $\Xi$ has to be volume minimizing in its homology class, i.e., special Lagrangian.

Recall that the concrete $\mathcal{N}=1$ subalgebra preserved by a $D 6$-brane wrapping the sLag $\Pi$ is determined by the phase $\theta$ appearing in

$$
\operatorname{Im}\left(\left.e^{i \pi \theta} \Omega\right|_{\Pi}\right)=0 .
$$

For $D 6$-branes the value $\theta=0 \bmod 2$ corresponds to the $\mathcal{N}=1$ algebra preserved also by the orientifold planes. Standard arguments taking into account the localization of the E2-brane in the external dimensions show that if it wraps a cycle $\Xi$ with $\theta_{\Xi}=0$ it preserves the supercharges $Q_{\alpha}, \bar{Q}_{\dot{\alpha}}^{\prime}$ and breaks $\bar{Q}_{\dot{\alpha}}, Q_{\alpha}^{\prime}$, where the unprimed and primed quantities $Q_{\alpha}, \bar{Q}_{\dot{\alpha}}$ and $Q_{\alpha}^{\prime}, \bar{Q}_{\dot{\alpha}}^{\prime}$ generate the 
$\mathcal{N}=1$ subalgebra preserved by the orientifold and the one orthogonal to it, respectively.

To restore supersymmetry in the topological sector containing the E2-brane we have to integrate all amplitudes over the corresponding Goldstone fermions $\bar{\theta}_{\dot{\alpha}}$ and $\theta_{\alpha}^{\prime}$ associated with the violation of $\bar{Q}_{\dot{\alpha}}$ and $Q_{\alpha}^{\prime}$. Depending on the details of the orientifold projection, the $\theta_{\alpha}^{\prime}$ can be projected out provided the cycle $\Xi$ is invariant, $\Xi=\Xi^{\prime}$ [21]. In this case, the associated topological sector contributes to the antiholomorphic superpotential involving the antichiral superfields. We identify it as the anti-instanton sector. By contrast, the instanton, given by $\theta_{\Xi}=1 \bmod 2$, preserves the supercharges $\bar{Q}_{\dot{\alpha}}, Q_{\alpha}^{\prime}$ and violates $Q_{\alpha}, \bar{Q}_{\dot{\alpha}}^{\prime}$, thus contributing to the holomorphic superpotential provided the $\bar{\theta}_{\dot{\alpha}}^{\prime}$ are projected out. This is the situation we are interested in when computing corrections to the superpotential.

In the sequel it will be useful to consider only “aligned" cycles $\Xi$ with $\theta_{\Xi}=0 \bmod 2$ wrapped by the (anti-)instanton. At the CFT level, the fact that the instanton is actually "antialigned" with respect to the D6-branes internally is taken into account by projecting the spectrum between the E2- and the D6-branes of the model onto its GSO-odd part. For the anti-instanton, we keep the GSOeven states. From the worldvolume perspective, the two objects clearly carry opposite charge under the RamondRamond (RR) three-form $C_{3}$ coupling to the worldvolume. The classical part of the Euclidean (anti-)instanton action appearing as $e^{-S_{E 2}}$ in corresponding F-term couplings reads

$$
S_{E 2}=\frac{2 \pi}{\ell_{s}^{3}}\left(\frac{1}{g_{s}} \mathrm{Vol}_{\Xi} \mp i \int_{\Xi} C_{3}\right),
$$

with the (lower) upper sign corresponding to (anti-)instantons. ${ }^{3}$

The zero modes of the (anti-)instanton can be computed in setups where the $\mathcal{N}=(2,2)$ CFT describing the internal sector is known exactly. The general form of the various vertex operators can be found in [1]. For the sake of concreteness and as preparation for our explicit computations, we specialize here to the case that the D6- and the E2-branes wrap factorizable three-cycles of toroidal orientifolds. $^{4}$

There are three different sectors to distinguish corresponding to the boundary conditions of the open strings.

\section{1. $E 2-E 2$}

This sector contains the usual four bosonic zero modes $x_{E}^{\mu}$ corresponding to the Goldstone bosons associated with the breakdown of four-dimensional Poincaré invariance

\footnotetext{
${ }^{3}$ We define the string length as $\ell_{s}=\sqrt{2 \pi \alpha^{\prime}}$.

${ }^{4}$ A detailed summary of the covariant open string quantization between two D6-branes in this context can be found, e.g., in Appendix A of [22].
}

due to the localization of the instanton in spacetime. Their vertex operators [in the $(-1)$ picture] are simply given by

$$
V_{x_{E}^{\mu}}(z)=\Omega_{E 2 E 2} x_{E}^{\mu} \frac{1}{\sqrt{2}} \psi_{\mu}(z) e^{-\varphi(z)} .
$$

Here $\Omega_{E 2 E 2}$ denotes the Chan-Paton factor. The polarization $x_{E}^{\mu}$ carries no mass dimension, corresponding to a field in $d=0$ dimensions. It is related to the position $x_{0}^{\mu}$ of the instanton in external spacetime [14] via $x_{E}^{\mu}=x_{0}^{\mu} / \ell_{s}$. The factor $1 / \sqrt{2}$ accounts for the fact that $\psi_{\mu}(z)$ are real fields. In this and all following vertex operators we absorb the open string coupling into the polarization (see Sec. II B for a detailed discussion of this point).

In general, the fermionic superpartners are given by four Weyl spinors $\theta_{\alpha}, \bar{\theta}_{\dot{\alpha}}$ with vertex operators [in the $(-1 / 2)$ picture]

$$
\begin{aligned}
& V_{\theta}(z)=\Omega_{E 2 E 2} \theta_{\alpha} S^{\alpha}(z) \prod_{I=1}^{3} e^{(i / 2) H_{I}(z)} e^{-\varphi(z) / 2}, \quad q=3 / 2, \\
& V_{\bar{\theta}}(z)=\Omega_{E 2 E 2} \bar{\theta}_{\dot{\alpha}} S^{\dot{\alpha}}(z) \prod_{I=1}^{3} e^{-(i / 2) H_{I}(z)} e^{-\varphi(z) / 2}, \quad q=-3 / 2 .
\end{aligned}
$$

Here $H_{I}(z)$ denotes the bosonization of the complexified internal fermions $\Psi^{I}$ and $S^{\alpha}\left(S^{\dot{\alpha}}\right)$ are the left(right-)handed four-dimensional spin fields. We have also included the world sheet charge $q$. The fermionic zero modes are to be identified with the four Goldstinos discussed above [in (6) and in the sequel we omit the primes for simplicity].

Clearly, all these states are even under the usual GSO projection given in the covariant formulation by

$$
\begin{gathered}
R:(-1)^{F}=(-i) \exp \left(i \pi \sum_{i=0}^{4} s_{i}\right), \\
N S:(-1)^{F}=(-1) \exp \left(i \pi \sum_{i=0}^{4} s_{i}\right),
\end{gathered}
$$

with $s_{i}= \pm 1 / 2$ and \pm 1 , respectively. As anticipated, in computing amplitudes we have to integrate over the Goldstone bosons $d^{4} x_{E}^{\mu}$ as well as all four Goldstinos $d^{2} \theta_{\alpha}$ and $d^{2} \bar{\theta}_{\dot{\alpha}}$ to restore four-dimensional Poincaré invariance and $\mathcal{N}=1$ supersymmetry. Only if the modes $\bar{\theta}_{\dot{\alpha}}\left(\theta_{\alpha}\right)$ are projected out [21] for (anti-)instantons will this result in superpotential couplings.

In general the $E 2-E 2$ sector also comprises $b_{1}(\Xi)$ chiral superfields corresponding to the position moduli of the E2-brane. On toroidal backgrounds, they are associated with the moduli along those two-tori in which the E2-brane is not fixed. For completeness, we display the vertex operators for the chiral component fields corresponding to the position moduli in the, say, first torus, 


$$
\begin{gathered}
V_{c}(z)=\Omega_{E 2 E 2} c e^{i H_{1}(z)} e^{-\varphi(z)} \\
V_{\chi_{\alpha}}=\Omega_{E 2 E 2} \chi_{\alpha} S^{\alpha}(z) e^{(i / 2) H_{1}(z)} \prod_{I=2,3} e^{-(i / 2) H_{I}(z)} e^{-\varphi(z) / 2}
\end{gathered}
$$

to be supplemented in general by their antichiral counterparts, again possibly modulo the issue of orientifold projections. The need to integrate, in particular, over the modulini yields nonvanishing instanton amplitudes only once they are absorbed by couplings to additional closed or open string fermionic modes. On toroidal orbifolds, this results in higher fermion interactions ${ }^{5}$ first discussed in the context of world sheet instantons for heterotic $(0,2)$ models in [16]. The corresponding effect for nonrigid heterotic five-branes has been studied in [24]. Being interested in contributions to the superpotential, we restrict ourselves to the study of instantons wrapping appropriate rigid threecycles $\Xi$ in the sequel.

\section{2. $E 2-D 6$}

Additional zero modes arise at the intersection of the $E 2$-brane with the various $D 6$-branes from open strings localized at the intersection point (or the overlap manifold). Open strings in this sector are subject to DirichletNeumann (DN) boundary conditions in the extended four dimensions and to mixed DN boundary conditions internally, depending on the concrete intersection angles. The external DN conditions shift the oscillator moding in these directions by $1 / 2$. In the Ramond sector, the zero point energy is still vanishing and we find massless fermions. The novelty as compared to the case of spacetime filling branes at angles is that the degeneracy of states is lifted in that the four-dimensional spin fields $S_{\alpha}$ or $S_{\dot{\alpha}}$ are no longer present. This also affects the details of the GSO projection. In the Neveu-Schwarz sector, the vacuum energy is zero only for completely parallel branes, which is the only situation with bosonic zero modes in the nonsingular geometric phase.

We first consider the case of nontrivial intersection of an instanton (anti-instanton) wrapping $\Xi$ and a stack of $N_{a}$ $D 6$-branes wrapping $\Pi_{a}$ in all three two-tori. It gives rise to $[\Xi \cap \Pi]^{+}$fermionic zero modes in the $\left(\mathbf{N}_{\mathrm{a}},-1_{E}\right)$ $\left(\left(1_{E}, \overline{\mathbf{N}}_{\mathbf{a}}\right)\right)$ and $[\Xi \cap \Pi]^{-}$fermionic zero modes in the respective conjugate representation [1].

To see this, the correct form of the vertex operators is needed. For actual computations it is indispensable to carefully distinguish between positive and negative inter-

\footnotetext{
${ }^{5}$ This is in agreement with the analysis in [23] of the superpotential induced by nonrigid supersymmetric membrane instantons on $G_{2}$ manifolds. The superpotential was found to be proportional to the Euler characteristic of the moduli space of the calibrated three-cycle wrapped by the instanton, which vanishes for nonrigid factorizable sLags on toroidal backgrounds.
}

section angles in the three two-tori. Generically, the intersection number between factorizable three-cycles $\Pi_{a}$ and $\Pi_{b}$ are given by

$$
I_{a b}=\prod_{I=1}^{3} I_{a b}^{I}
$$

where $I_{a b}^{I}$ denotes the intersection number in the $I$ th torus. Here positive (negative) intersection number $I_{a b}^{I}$ corresponds to positive (negative) angle $\theta_{a b}^{I}$ and it is understood that $\left|\theta_{a b}^{I}\right|<1$.

Given the total intersection number, say $I_{a b}>0$, one distinguishes four different cases, three cases where one has negative intersection number $I_{a b}^{I}$ in two internal tori and positive in the left one and the symmetric one in which the intersection numbers in all three internal two-tori are positive. In supersymmetric configurations the intersection angles add up to 2 for the latter choice, while for the other three their sum is 0 .

Consider now an instanton wrapping the cycle $\Xi$ such that all intersection angles $\theta_{E 2 a}^{I}$ are positive for some cycle $\Pi_{a}$ wrapped by a $D 6$-brane. Upon projection onto states odd under the GSO operator (7), the vertex operator for the fermionic zero mode $\lambda_{a}$ at the intersection $E 2-a$ is given by

$$
V_{\lambda_{a}}=\Omega_{a E 2} \lambda_{a} \Sigma(z) \prod_{I=1}^{3} \sigma_{1-\theta_{E 2 a}^{I}}(z) e^{-i\left(\theta_{E 2 a}^{I}-1 / 2\right) H_{I}(z)} e^{-\varphi(z) / 2}
$$

Here $\Sigma(z)$ denotes the bosonic twist field ensuring Dirichlet-Neumann boundary conditions in spacetime. The $\lambda_{a}$ are Grassmannian variables and represent the polarization of the fermionic zero mode, normalized again as a field in $D=0$ dimensions. Note that the GSO projection forces us to keep only the state in the sector starting from the D6-brane and ending on the E2 and projects out the state with the reversed orientation. The relevant intersection angles are therefore negative and lead to the above form of the vertex (see, e.g., [22]) carrying world sheet charge $q=-1 / 2$. As indicated by the $C P$ indices, it transforms as $\left(\mathbf{N}_{\mathbf{a}},-1_{E}\right)$.

For anti-instantons, we have to keep the state oriented from $E 2$ to $D 6$ [i.e., transforming as $\left(1_{E}, \overline{\mathbf{N}}_{\mathbf{a}}\right)$ ] and of world sheet charge $q=1 / 2$. We will refer to states negatively charged under $U(1)_{a}$ as $\bar{\lambda}_{a}$. The various remaining cases are dealt with analogously. This finally leads to the index theorem stated above.

For completeness, we briefly discuss nonchiral intersections between the $E 2$ - and the $D 6$-branes. Consider first the supersymmetric situation that the corresponding cycles are parallel in one torus such that, say, $\theta_{E 2 a}^{1}>0, \theta_{E 2 a}^{2}=$ $-\theta_{E 2 a}^{1}, \theta_{E 2 a}^{3}=0$. For instantons, we find one chiral fermionic zero mode in the $E 2 \rightarrow D 6_{a}$ sector with vertex 


$$
\begin{aligned}
V_{\bar{\lambda}_{a}}= & \Omega_{E 2 a} \bar{\lambda}_{a} \Sigma(z) \sigma_{\theta_{E 2 a}^{1}}(z) e^{i\left(\theta_{E 2 a}^{1}-1 / 2\right) H_{1}(z)} \sigma_{1-\theta_{E 2 a}^{1}}(z) \\
& \times e^{i\left(-\theta_{E 2 a}^{1}+1 / 2\right) H_{2}(z)} e^{-(i / 2) H_{3}(z)} e^{-(\varphi(z) / 2)}
\end{aligned}
$$

and one in the $D 6_{a} \rightarrow E 2$ sector with vertex

$$
\begin{aligned}
V_{\lambda_{a}}= & \Omega_{a E 2} \lambda_{a} \Sigma(z) \sigma_{1-\theta_{E 2 a}^{1}}(z) e^{i\left(-\theta_{E 2 a}^{1}+1 / 2\right) H_{1}(z)} \sigma_{\theta_{E 2 a}^{1}}(z) \\
& \times e^{i\left(\theta_{E 2 a}^{1}-1 / 2\right) H_{2}(z)} e^{-(i / 2) H_{3}(z)} e^{-(\varphi(z) / 2)} .
\end{aligned}
$$

Note that both zero modes carry world sheet charge $q=$ $-1 / 2$, i.e., are "chiral" from the world sheet point of view. The corresponding modes for anti-instantons should be clear.

If finally the E2- and D6-branes are completely parallel internally, the fermionic instanton zero-mode sector for nonrigid cycles comprises simply the four states

$$
V_{\lambda_{a}}=\Omega_{a E 2} \lambda_{a} \Sigma(z) \prod_{I=1}^{3} e^{i S_{I} H_{I}(z)} e^{-\varphi(z) / 2}
$$

with world sheet charge $q=\sum_{I} s_{I}=3 / 2$ or $-1 / 2$ and likewise four $\bar{\lambda}_{a}$ in the $E 2 \rightarrow D 6_{a}$ sector. Note that for completely rigid branes only the two states $q=3 / 2$ are present. Since the zero point energy vanishes for completely trivial intersections, the lowest lying bosons are now also massless. In both the $E 2 \rightarrow D 6_{a}$ and the $E 2 \rightarrow$ $D 6_{a}$ sector the GSO projection removes 2 out of the 4 spinorial ground states from the external dimensions, leaving for instantons

$$
V_{w_{\dot{\alpha}}}=\Omega_{a E 2} w_{\dot{\alpha}} S^{\dot{\alpha}}(z) \Sigma(z) e^{-\varphi(z)}
$$

(plus the orientation reversed one), whereas the antiinstanton carries chiral modes.

\section{3. $E 2-E 2^{\prime}$}

In general, there are additional zero modes at the intersection of the E2-brane and its orientifold image. Because of the Dirichlet-Dirichlet boundary conditions, the orientifold projection picks up an additional minus sign as compared to the $D 6-D 6^{\prime}$ sector. For single instantons, we therefore find $\frac{1}{2}\left(I_{E 2 E 2^{\prime}}+I_{O 6 E 2}\right)$ bosonic and/or fermionic zero modes carrying charge 2 under $U(1)_{E 2}$. Their vertex operators are identical to the more familiar massless states between $D 6$-branes and their images. For a straightforward generation of actual superpotential terms we insist that these modes be absent.

\section{B. Amplitudes - generalities and normalization}

E2-instantons of the above kind can induce F-term couplings involving the open string superfields $\Phi_{a b}$ between the D6-branes present in the model. Of particular interest are those couplings which are absent perturbatively since they violate some of the global Abelian symmetries which are the remnants of the $U(1)$ gauge symmetries on the D6-branes broken by Stückelberg-type couplings to the $\mathrm{RR}$ forms of the background. The exponential suppression factor $e^{-S_{E 2}}$ characteristic for instantonic couplings transforms under the global $U(1)$ symmetries in such a way that the full coupling

$$
W_{n p}=\prod_{i} \Phi_{a_{i} b_{i}} e^{-S_{E 2}}
$$

is invariant again. More precisely, from the axionic shift symmetries under these Abelian symmetries induced by the Chern-Simons couplings of the $N_{a} D 6_{a}$-branes one finds the $U(1)_{a}$ transformation of the instanton [1] (see also [3]),

$$
\begin{aligned}
e^{-S_{E 2}} & =\exp \left[\frac{2 \pi}{\ell_{s}^{3}}\left(-\frac{1}{g_{s}} \mathrm{Vol}_{\Xi}+i \int_{\Xi} C^{(3)}\right)\right] \\
& \rightarrow e^{i Q_{a}(E 2) \Lambda_{a}} e^{-S_{E 2}}
\end{aligned}
$$

with

$$
Q_{a}(E 2)=N_{a} \Xi \circ\left(\Pi_{a}-\Pi_{a}^{\prime}\right) .
$$

Indeed this charge is exactly the amount of $U(1)_{a}$ charge carried by the fermionic zero modes between the $E 2$ and the $D 6_{a}$, which serves as an important check that our identification of the instanton versus anti-instanton and the associated choice of GSO projection is correct.

The general procedure for the computation of the instanton induced physical $M$-point couplings involving the canonically normalized fields of the four-dimensional effective action has been outlined in [1]. In momentum space it is given, after some refinements, by

$$
\begin{aligned}
& \left\langle\Phi_{a_{1}, b_{1}}\left(p_{1}\right) \cdot \ldots \cdot \Phi_{a_{M}, b_{M}}\left(p_{M}\right)\right\rangle_{E 2 \text {-inst }} \\
& =-\frac{1}{C} \int d^{4} \tilde{x}_{E} d^{2} \tilde{\theta} \sum_{\operatorname{conf}} \prod_{a}\left(\prod_{i=1}^{\left[\Xi n \Pi_{a}\right]^{+}} d \tilde{\lambda}_{a}^{i}\right) \\
& \quad \times\left(\prod_{i=1}^{\left[\Xi n \Pi_{a}\right]^{-}} d \tilde{\bar{\lambda}}_{a}^{i}\right) e^{-S_{E 2}} \times e^{Z_{0}^{\prime}} \times\left\langle\hat{\Phi}_{a_{1}, b_{1}}\left[\vec{x}_{1}\right]\right\rangle_{\lambda_{a_{1}}, \bar{\lambda}_{b_{1}}} \\
& \quad \ldots \cdot\left\langle\hat{\Phi}_{a_{L}, b_{L}}\left[\vec{x}_{L}\right]\right\rangle_{\lambda_{a_{L}}, \bar{\lambda}_{b_{L}}} \times \prod_{k}\left\langle\hat{\Phi}_{c_{k}, c_{k}}\left[\vec{x}_{k}\right]\right\rangle_{A\left(E 2, D 6_{c_{k}}\right.}^{\text {loop }} .
\end{aligned}
$$

Its basic building blocks are disk and annulus diagrams with insertion of an appropriate product of boundary changing vertex operators, denoted schematically by $\hat{\Phi}_{a_{1}, b_{1}}\left[\vec{x}_{1}\right]$. It is understood that either precisely two of these diagrams carry one $\theta$ mode each or one of them carries both. Each disk carries two of the fermionic modes $\lambda_{a}$ from the $E 2-D 6$ sector so that each combination $\lambda_{a_{i}} \hat{\Phi}_{a_{i}, b_{i}} \bar{\lambda}_{b_{i}}$ is gauge invariant, whereas the annulus diagrams are uncharged in that they are free of $\lambda_{a}$ insertions. The instanton suppression factor $e^{-S_{E 2}}$ arises from exponentiation of tree-level disks with no matter insertion and is corrected by the exponentiated regularized one-loop amplitude $e^{Z^{\prime}}$ with 


$$
Z^{\prime}=\sum_{a}\left[\mathcal{A}\left(D 6_{a}, E 2\right)+\mathcal{A}\left(D 6_{a}^{\prime}, E 2\right)\right]+\mathcal{M}(E 2, O 6)
$$

in terms of the annulus and Möbius amplitudes $\mathcal{A}$ and $\mathcal{M}$ (for details see [1]). This one-loop factor has been computed in $[6,7]$ and is related to the regularized threshold correction to the gauge coupling of a D6-brane wrapping the same internal cycle $\Xi$ as the instanton. The latter have been determined in [25] for toroidal orientifolds. The combinatorical prefactor $-\frac{1}{C}$ arises from expansion of the exponentiated instanton moduli action $e^{-S_{\bmod }}$ containing the tree-level and annulus coupling terms.

After this review we clarify the proper $g_{s}$ normalization of the instanton amplitude. It is convenient to work in the frame where all vertex operators (including the ones for fields between two $D 6$-branes) carry no explicit factors of $g_{s}$. A disk with boundary only on one type of $D p$-brane carries the normalization factor

$$
C_{p}=\frac{2 \pi}{g_{s} \ell_{s}^{p+1}}
$$

Consequently, all kinetic and tree-level perturbative coupling terms arise formally at order $g_{s}^{-1}$. This is therefore the tree-level order in $g_{s}$ to which nonperturbative couplings are to be compared.

The disks appearing in the above expression (17) are bounded partly by the $D 6$-branes $a$ and $b$ and the E2-instanton. In such a case, the amplitude has to be normalized with respect to the dimension of the overlap of the branes involved and therefore carries a factor (see also [6])

$$
C=\frac{2 \pi}{g_{s}} .
$$

Consider now the normalization of the instanton moduli measure. As noted, the integration over the fourdimensional supermoduli $\int d^{4} \tilde{x}_{E} d^{2} \tilde{\theta}$ restores Poincaré invariance and $\mathcal{N}=1$ supersymmetry. The inclusion of the charged zero modes $\tilde{\lambda}_{a}$ in the measure can be understood as the process of integrating these modes out since they would result in a zero in the Pfaffian $e^{Z^{\prime}}$ [26]. While the Grassmannian integral is trivial and merely results in a combinatorical factor, the integration over $d^{4} \tilde{x}_{E}$ will ensure momentum conservation of the $M$-point amplitude [see Eq. (35)]. The tilde indicates that we have to integrate over the properly normalized zero modes corresponding to the instanton moduli in the ADHM action in the limit where the E2-brane wraps the same cycle as one of the $D 6$-branes and therefore represents a gauge instanton (or its stringy generalization for $\ell_{s} \neq 0$ ). The resulting Jacobian in the transition from the polarizations appearing in our vertex operators takes care of the proper normaliza- tion procedure in the more familiar case of field theory instantons (see, e.g., the review [27] for details). The situation of parallel E2- and D6-branes is T-dual to the $D(3)-D(-1)$ system in type IIB theory. Adapting the CFT analysis of [15] to our case, ${ }^{6}$ we find the following relation between the polarization in the vertices and the ones to appear in the measure,

$$
\begin{gathered}
\tilde{x}_{E}^{\mu}=\frac{x_{E}^{\mu}}{2} \sqrt{\frac{2 \pi \bar{V}_{E 2}}{g_{s}}}, \quad \tilde{\theta}^{\alpha}=\theta^{\alpha} \sqrt{\frac{2 \pi \mathcal{V}_{E 2}}{g_{s}}} \\
\tilde{\lambda}=\lambda \sqrt{\frac{2 \pi}{g_{s}}},
\end{gathered}
$$

where $\mathcal{V}_{E 2}=\operatorname{Vol}_{E 2} / \ell_{s}^{3}$. Most importantly, the contribution from two $\lambda$ modes $^{7}$ cancels the $g_{s}$-dependent topological normalization of the disk (19).

If indeed precisely $2 \lambda$ modes are inserted per disk (and none on the annulus diagrams carrying an additional factor of $g_{s}$ in their normalization), then the induced M-point amplitude is proportional to $2 \pi \mathcal{V}_{E 2} / g_{s}$ due to the remaining normalization factors from $\int d^{4} \tilde{x}_{E} d^{2} \tilde{\theta}$ (times the exponential dependence, of course). It therefore arises at "string tree level" as compared to the perturbative terms.

\section{Amplitudes-CFT details}

A phenomenologically interesting application is the computation of instanton induced $U(1)$ charge violating 2-point couplings. These can be thought of as Majorana masses for right-handed neutrinos or as $\mu$ terms in the MSSM [1,3,5]. We will now compute such 2-point couplings in a general setup, which can then be adapted to concrete examples.

Consider the superfield $\Phi_{a b}^{A}$ at the intersection $A$ between two D6-branes wrapping the cycles $\Pi_{a}$ and $\Pi_{b}$. We would like to generate couplings of the form $\left\langle\psi_{a b}^{A} \psi_{a b}^{B}\right\rangle_{E 2}$. The zero-mode structure of the instanton has to allow for a compensation of the excess of $U(1)_{a}$ and $U(1)_{b}$ charge. This requires

$$
\begin{array}{lll}
{\left[\Pi_{E 2} \cap \Pi_{a}\right]^{+}=2} & {\left[\Pi_{E 2} \cap \Pi_{b}\right]^{-}=2} & \text { for } I_{a b}>0 \\
{\left[\Pi_{E 2} \cap \Pi_{a}\right]^{-}=2} & {\left[\Pi_{E 2} \cap \Pi_{b}\right]^{+}=2} & \text { for } I_{a b}<0
\end{array}
$$

and the intersection between the E2- and all other

\footnotetext{
${ }^{6}$ Unlike [15] we do not assign four-dimensional canonical mass dimensions to the instanton moduli but treat them as dimensionless fields in zero dimensions. The disk normalization between parallel $E 2$ - and $D 6$-branes is $2 \pi V_{E 2} / g_{s}$. The resulting amplitudes and effective moduli action before rescaling therefore differ by a power of $V_{E 2} / \ell_{s}^{4}$ as compared to the ones in [15]. Our rescaling for the case of parallel $E 2$ and $D 6$ systems is otherwise identical upon replacing $g_{0} \rightarrow \sqrt{g_{s} V_{E 2} / \pi}$. Finally, for E2- and D6-branes at angles, the rescaling of the $\lambda$ modes does not contain any $\mathcal{V}_{E 2}$, in agreement with (19).

${ }^{7}$ Recall that $d(a \psi)=a^{-1} d \psi$ for a Grassmann field $\psi$.
} 
D6-branes has to vanish. ${ }^{8}$ We reiterate that the absence of additional reparametrization and other uncharged zero modes in the $E 2-E 2^{\prime}$ sector necessitates the E2-brane to be rigid and to satisfy $\left[\Pi_{E 2} \cap \Pi_{E 2^{\prime}}\right]^{ \pm}=0$. The four zero modes are denoted by $\lambda_{a}^{i}$ and $\bar{\lambda}_{b}^{k}$ for $i, k=1,2$. Since the CFT computation depends on the concrete form of the vertex operators, we have to make a definite choice of angles and intersection numbers. Consider, e.g., the simple situation corresponding to $I_{a b}>0$ such that ${ }^{9}$

$$
\begin{aligned}
& \theta_{a b}^{I}>0, \quad \theta_{E 2 a}^{I}>0, \quad \theta_{E 2 b}^{I}<0, \\
& \sum_{I=1}^{3} \theta_{a b}^{I}=\sum_{I=1}^{3} \theta_{E 2 a}^{I}=2=-\sum_{I=1}^{3} \theta_{E 2 b}^{I} .
\end{aligned}
$$

With this choice of angles the vertex operator for $\psi_{a b}^{A}$ takes the form

$$
\begin{aligned}
V_{\psi_{a b}^{A}}= & \ell_{s}^{3 / 2} \Omega_{b a} \psi_{\alpha}^{A} S^{\alpha}(z) \prod_{I=1}^{3} \sigma_{1-\theta_{a b}^{I}}(z) e^{-i\left(\theta_{a b}^{I}-1 / 2\right) H_{I}(z)} \\
& \times e^{i k_{\mu}^{A} X^{\mu}(z)} e^{-\varphi(z) / 2}
\end{aligned}
$$

where $\psi_{\alpha}^{A}$ carries canonical mass dimensions. The vertex for the zero mode at the intersection of $E 2$ and $D 6_{a}$ has been given in (11), and the one between $E 2$ and $D 6_{b}$ reads

$$
V_{\bar{\lambda}_{b}^{k}}=\Omega_{E 2 b} \bar{\lambda}_{b}^{k} \Sigma(z) \prod_{I=1}^{3} \sigma_{1+\theta_{E 2 b}^{I}}(z) e^{i\left(\theta_{E 2 b}^{I}+1 / 2\right) H_{I}(z)} e^{-\varphi(z) / 2} .
$$

We then have to compute

$$
\begin{aligned}
\left\langle\psi_{a b}^{A} \psi_{a b}^{B}\right\rangle_{E 2}= & -\frac{1}{2 !} \frac{V_{E 2}}{16} \frac{g_{s}}{2 \pi} \int d^{4} x_{E} \int d^{2} \theta \int d^{2} \lambda_{a} \\
& \times \int d^{2} \bar{\lambda}_{b} e^{-S_{\text {inst. }} e^{Z^{\prime}}} \\
& \times \sum_{i, j, k, l}\left\langle V_{\Theta^{\alpha}}^{-1 / 2} V_{\bar{\lambda}_{b}^{k}}^{-(1 / 2)} V_{\psi_{\alpha}^{A}}^{-(1 / 2)} V_{\lambda_{a}^{i}}^{-(1 / 2)}\right\rangle \\
& \times\left\langle V_{\Theta^{\beta}}^{-(1 / 2)} V_{\bar{\lambda}_{b}^{l}}^{-(1 / 2)} V_{\psi_{\beta}^{B}}^{-(1 / 2)} V_{\lambda_{a}^{j}}^{-(1 / 2)}\right\rangle .
\end{aligned}
$$

This already includes the rescaling [20]. It is understood that the summation is only over those combinations of family indices with nontrivial disk diagrams. This important point has to be studied in concrete examples.

The disk amplitudes appearing in (25) can be evaluated using standard CFT methods. The computation of the fourpoint function $\langle\theta \bar{\lambda} \psi \lambda\rangle$ involving the vertex operators (6), (11), (23), and (24) requires the following correlators

\footnotetext{
${ }^{8}$ Strictly speaking, this is only true if the $E 2$ lies away from the orientifold brane. In case $E 2=E 2^{\prime}$, the $E 2-a$ and $a^{\prime}-E 2$ sectors are identified.

${ }^{9}$ Note that in most concrete realizations including our example given in Sec. III the angles will be less symmetric, but this can easily be dealt with.
}

$$
\begin{aligned}
\left\langle\prod_{i=1}^{4} e^{-\varphi\left(z_{i}\right) / 2}\right\rangle & =\prod_{i=1}^{4} z_{i j}^{-1 / 4}, \\
\left\langle S^{\alpha}\left(z_{1}\right) S^{\beta}\left(z_{2}\right)\right\rangle & =\epsilon^{\alpha \beta} z_{12}^{-1 / 2}, \\
\left\langle e^{i \alpha H_{I}\left(z_{1}\right)} e^{i \beta H_{I}\left(z_{2}\right)} e^{i \gamma H_{I}\left(z_{3}\right)} e^{i \delta H_{I}\left(z_{4}\right)}\right\rangle & =z_{12}^{\alpha \beta} z_{13}^{\alpha \gamma} z_{14}^{\alpha \delta} z_{23}^{\beta \gamma} z_{24}^{\beta \delta} z_{34}^{\gamma \delta}, \\
\left\langle\Sigma\left(z_{1}\right) e^{i k_{\mu} X^{\mu}\left(z_{2}\right)} \Sigma\left(z_{3}\right)\right\rangle & =e^{i k_{\mu} x_{0}^{\mu}} z_{13}^{-1 / 2} .
\end{aligned}
$$

Here $z_{i j}$ denotes $z_{i}-z_{j}$ and $x_{0}$ is the position of the $E 2$-instanton in spacetime. The most involved ingredient is the correlator of the three bosonic twist fields. In general, it reads $[28,29]$

$$
\begin{aligned}
& \left\langle\sigma_{\alpha}\left(z_{1}\right) \sigma_{\beta}\left(z_{2}\right) \sigma_{\gamma}\left(z_{3}\right)\right\rangle \\
& \quad=\left(4 \pi \Gamma_{\alpha, \beta, \gamma}\right)^{1 / 4} z_{12}^{-\alpha \beta} z_{13}^{-\alpha \gamma} z_{23}^{-\beta \gamma} \sum_{m} e^{-\mathcal{A}(m),}
\end{aligned}
$$

where $\Gamma_{\alpha, \beta, \gamma}$ is given by

$$
\Gamma_{\alpha, \beta, \gamma}=\frac{\Gamma(1-\alpha) \Gamma(1-\beta) \Gamma(1-\gamma)}{\Gamma(\alpha) \Gamma(\beta) \Gamma(\gamma)}
$$

and $\mathcal{A}(m)=A(m) /\left(2 \pi \alpha^{\prime}\right)$ is the area in string units of the triangle formed by the three intersecting branes. The correlator (27) vanishes if the angles $\alpha, \beta, \gamma$ do not add up to an integer. The normalization $(4 \pi)^{1 / 4}$ was determined in [28] by factorizing the four-point amplitude involving four bosonic twist fields in the limit corresponding to a gauge boson exchange.

Upon including the disk normalization factor $2 \pi / g_{s}$ and using the supersymmetry conditions (22), we find

$$
\begin{aligned}
\left\langle\theta^{\alpha} \bar{\lambda}_{b}^{k} \psi_{\alpha}^{A} \lambda_{a}^{i}\right\rangle= & \frac{2 \pi \ell_{s}^{3 / 2}}{g_{s}} \operatorname{Tr}\left(\Omega_{E 2 E 2} \Omega_{E 2 b} \Omega_{b a} \Omega_{a E 2}\right) \\
& \times \theta^{\alpha} \bar{\lambda}_{b}^{k} \psi_{\alpha}^{A} \lambda_{a}^{i} \prod_{I=1}^{3}\left[4 \pi \Gamma_{\left.1-\theta_{a b}^{I}, 1-\theta_{E 2 a^{I}}, 1+\theta_{E 2 b}^{I}\right]^{1 / 4}}\right. \\
& \times \sum_{m_{I}} e^{-\mathcal{A}_{i k}^{A}\left(m_{I}\right)} \int \frac{\prod_{i=1}^{4} d z_{i}}{V_{C K G}} z_{13}^{-1 / 2} z_{24}^{-1 / 2} \\
& \times \prod_{i, j=1}^{4} z_{i j}^{-1 / 2} e^{-i k_{\mu}^{A} x_{0}^{\mu}}
\end{aligned}
$$

After we fix the vertex operator positions to ${ }^{10}$

$$
z_{1}=0, \quad z_{2}=x, \quad z_{3}=1, \quad z_{4}=\infty
$$

and add the c-ghost part

$$
\left\langle c\left(z_{1}\right) c\left(z_{3}\right) c\left(z_{4}\right)\right\rangle=z_{13} z_{14} z_{34}
$$

the amplitude computes to ${ }^{11}$

\footnotetext{
${ }^{10} \mathrm{We}$ need to include the other cyclic order as well.

${ }^{11}$ Note that even after taking into account the other cyclic order the only nonvanishing trace is $\operatorname{Tr}\left(\Omega_{E 2 E 2} \Omega_{E 2 b} \Omega_{b a} \Omega_{a E 2}\right)$ and therefore we need to integrate from 0 to 1 .
} 


$$
\left\langle\theta^{\alpha} \bar{\lambda}_{b}^{k} \psi_{\alpha}^{A} \lambda_{a}^{i}\right\rangle=\frac{2 \pi}{g_{s}} \ell_{s}^{3 / 2} C_{i k}^{A} e^{-i k_{\mu}^{A} x_{0}^{\mu}}\left(\theta^{\alpha} \bar{\lambda}_{b}^{k} \psi_{\alpha}^{A} \lambda_{a}^{i}\right)
$$

with

$$
C_{i k}^{A}=\pi \prod_{I=1}^{3}\left[4 \pi \Gamma_{1-\theta_{a b}^{I}, 1-\theta_{E 2 a^{I}}^{I}, 1+\theta_{E 2 b}^{I}}\right]^{1 / 4} \sum_{m_{I}} e^{-\mathcal{A}_{i k}^{A}\left(m_{I}\right)} .
$$

Here we omit the trivial trace structure and use

$$
\int_{0}^{1} d x[x(1-x)]^{-1 / 2}=\pi
$$

In order to obtain the coupling $\left\langle\psi_{a b}^{A} \psi_{a b}^{B}\right\rangle_{E 2}$ we plug (32) into (25) and perform the integrals over all fermionic and bosonic zero modes. In doing so, we make use of the integral representation of the $\delta$ function (recall that $x_{0}^{\mu}=$ $\left.\ell_{s} x_{E}^{\mu}\right)$

$$
\int d^{4} x_{E} e^{-i k_{\mu} x_{0}^{\mu}}=\frac{(2 \pi)^{4}}{\ell_{s}^{4}} \delta^{4}(k)
$$

and find

$$
\begin{aligned}
\left\langle\psi_{a b}^{A} \psi_{a b}^{B}\right\rangle_{E 2}= & -\frac{\mathcal{V}_{E 2} \pi}{16 g_{s}} M_{s} e^{-S_{\text {inst }}} e^{Z^{\prime}} \psi_{\alpha}^{A} \epsilon^{\alpha \beta} \psi_{\beta}^{B}(2 \pi)^{4} \\
& \times \delta^{4}\left(k^{A}+k^{B}\right) \sum_{i, j, k, l} \epsilon^{i j} \epsilon^{k l} C_{i k}^{A} C_{j l}^{B} .
\end{aligned}
$$

The overall sign can always be absorbed into phases of the fermions. Note that due to the Grassmannian integral, nonvanishing mass terms occur only for a suitable family structure such that indeed $\sum_{i, j, k, l} \epsilon^{i j} \epsilon^{k l} C_{i k}^{A} C_{j l}^{B} \neq 0$.

\section{Family structure for (off-)diagonal bilinears in a simple example}

To appreciate this latter point, consider the family structure depicted schematically in Fig. 1. We can think of it as representing one of the three two-tori of a toroidal orientifold model with factorizable $D 6$-branes wrapping a onecycle on each torus. In this case, branes $a$ and $b$ correspond to wrapping numbers $(1,-2)$ and $(1,2)$, respectively. For simplicity we assume here that the complete family replication is due to multiple intersections on just the depicted torus, though more general situations leading to nonvanishing terms on a factorizable $T^{6}$ are possible. ${ }^{12}$ Consider now the instanton wrapping the cycle $(1,0)$.

Straightforward inspection of the possible triangles connecting the various intersection points reveals that the coupling terms in the (zero-dimensional) moduli action of the instanton are proportional to

\footnotetext{
${ }^{12}$ In fact, our local model discussed in the next section is more general in this respect.
}

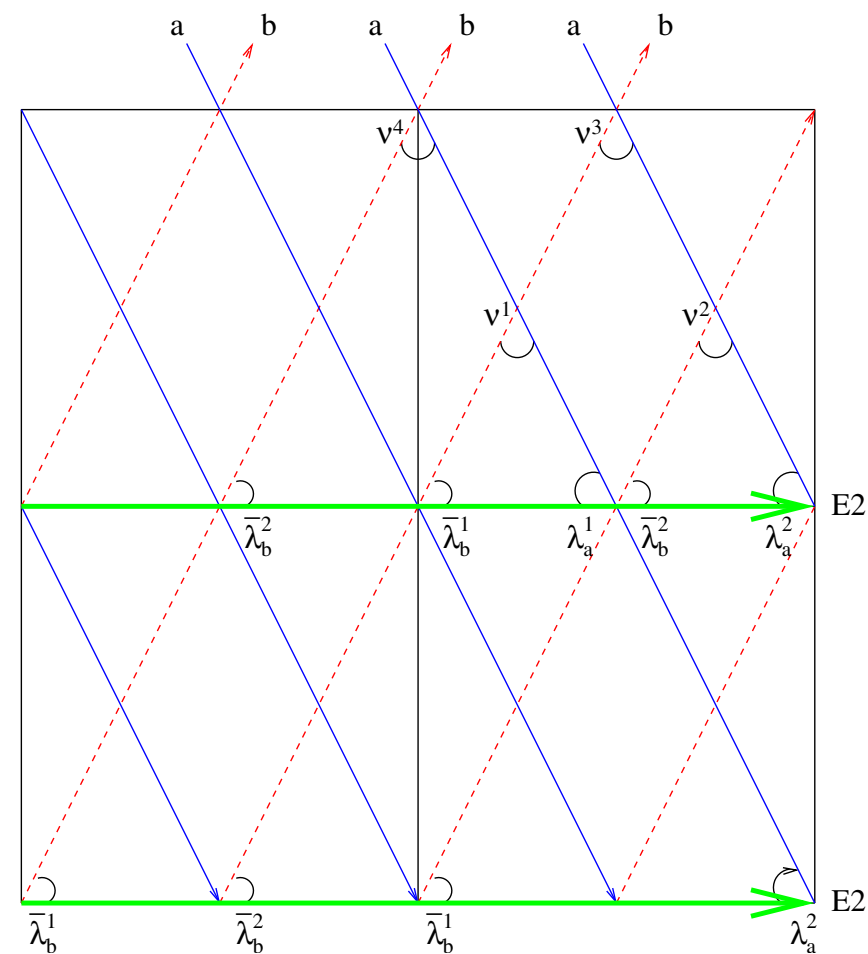

FIG. 1 (color online). Example of family structure yielding diagonal and off-diagonal fermion bilinears.

$$
\begin{aligned}
& \nu^{1} \theta \bar{\lambda}_{b}^{1} \lambda_{a}^{1} e^{-\mathcal{A}_{11}^{1}}+\nu^{1} \theta \bar{\lambda}_{b}^{2} \lambda_{a}^{2} e^{-\mathcal{A}_{22}^{1}}+\nu^{2} \theta \bar{\lambda}_{b}^{2} \lambda_{a}^{2} e^{-\mathcal{A}_{22}^{2}} \\
& +\nu^{2} \theta \bar{\lambda}_{b}^{1} \lambda_{a}^{1} e^{-\mathcal{A}_{11}^{2}}+\nu^{3} \theta \bar{\lambda}_{b}^{2} \lambda_{a}^{1} e^{-\mathcal{A}_{21}^{3}}+\nu^{3} \theta \bar{\lambda}_{b}^{1} \lambda_{a}^{2} e^{-\mathcal{A}_{12}^{3}} \\
& +\nu^{4} \theta \bar{\lambda}_{b}^{1} \lambda_{a}^{2} e^{-\mathcal{A}_{12}^{4}}+\nu^{4} \theta \bar{\lambda}_{b}^{2} \lambda_{a}^{1} e^{-\mathcal{A}_{21}^{4}} .
\end{aligned}
$$

Note that we have only given the leading area suppression due to the smallest possible triangles. The Grassmann integration now dictates which combinations of fermion bilinears can possibly appear in the amplitude. This results in the following fermion bilinears in the four-dimensional effective action,

$$
\begin{aligned}
S_{\text {nonpert }}= & -\int d^{4} x \frac{2 \pi}{g_{s}} M_{s} e^{-S_{\text {inst }}} e^{Z^{\prime}}\left(\nu^{1} \nu^{2} \nu^{3} \nu^{4}\right) \\
& \times \mathcal{M}\left(\nu^{1} \nu^{2} \nu^{3} \nu^{4}\right)^{T}
\end{aligned}
$$

with the $4 \times 4$ matrix

$$
\mathcal{M}=\left(\begin{array}{cc}
A & 0 \\
0 & B
\end{array}\right)
$$

given in terms of

$$
\begin{gathered}
A=\frac{\pi^{2}}{16}(4 \pi \Gamma)^{1 / 2}\left(\begin{array}{cc}
e^{-(\alpha+\beta)} & \frac{1}{2}\left(e^{-2 \alpha}+e^{-2 \beta}\right) \\
\frac{1}{2}\left(e^{-2 \alpha}+e^{-2 \beta}\right) & e^{-(\alpha+\beta)}
\end{array}\right), \\
B=\frac{\pi^{2}}{16}(4 \pi \Gamma)^{1 / 2}\left(\begin{array}{cc}
e^{-(\gamma+\delta)} & \frac{1}{2}\left(e^{-2 \gamma}+e^{-2 \delta}\right) \\
\frac{1}{2}\left(e^{-2 \gamma}+e^{-2 \delta}\right) & e^{-(\gamma+\delta)}
\end{array}\right) .
\end{gathered}
$$

Here we have defined 


$$
\begin{array}{ll}
\mathcal{A}_{11}^{1}=\mathcal{A}_{22}^{2}=\alpha, & \mathcal{A}_{22}^{1}=\mathcal{A}_{11}^{2}=\beta, \\
\mathcal{A}_{21}^{3}=\mathcal{A}_{12}^{4}=\gamma, & \mathcal{A}_{12}^{3}=\mathcal{A}_{21}^{4}=\delta
\end{array}
$$

and we have omitted the $(2 \pi)^{4} \delta\left(\sum k\right)$ in going from momentum to position-space. We have also made use of our freedom to absorb a phase $e^{i \pi / 2}$ into the fields $\nu^{1}$ and $\nu^{2}$ to adjust the signs of the Majorana mass terms.

As a result, we have found both diagonal couplings $\nu^{i} \nu^{i}$ and the off-diagonal ones $\nu^{1} \nu^{2}$ and $\nu^{3} \nu^{4}$. The overall scale of these terms is governed by the exponential suppression factor $e^{-S_{\text {inst }}}$, whereas the relative size of the various couplings is set by the ratio of the triangles involved. These depend on the concrete Kähler and open string moduli. For example, for a particular choice of brane positions we can set one of the areas, say $\alpha$, to zero, in which case the offdiagonal coupling $\nu^{1} \nu^{2}$ would dominate over the diagonal ones $\nu^{1} \nu^{1}, \nu^{2} \nu^{2}$.

Finally we point out that the above nonperturbative couplings in this example are allowed since not all possible intersection points are connected by world sheet instantons, i.e., disk triangles. As observed already in [30], this is a generic consequence of the fact that the three intersection numbers $I_{E a}, I_{E b}, I_{a b}$ are not coprime. If, by contrast, in addition to the couplings (37), also the combination, say,

$$
\nu^{1} \theta \bar{\lambda}_{a}^{1} \lambda_{b}^{2} e^{-\tilde{\mathcal{A}}_{12}^{1}}+\nu^{1} \theta \bar{\lambda}_{b}^{2} \lambda_{a}^{1} e^{-\tilde{\mathcal{A}}_{21}^{1}}
$$

were present, the Grassmann integral would give zero for the coupling $\nu^{1} \nu^{1}$ whenever $\tilde{\mathcal{A}}_{11}^{1}+\tilde{\mathcal{A}}_{22}^{1}=\mathcal{A}_{12}^{1}+\mathcal{A}_{21}^{1}$. This results in yet another important constraint on the architecture of concrete models exhibiting E2-instanton effects, as has also been addressed in [3].

\section{NONPERTURBATIVE MAJORANA MASSES IN A LOCAL GUT-LIKE BRANE SETUP}

In this section we present a local brane configuration on the orientifold $T^{6} / \mathbb{Z}_{2} \times \mathbb{Z}_{2}^{\prime}$ which serves as a toy model for realizing the seesaw mechanism for neutrino masses. While our ultimate object of desire are globally consistent MSSM-like string vacua satisfying all tadpole- and Ktheory constraints, we content ourselves for the time being with a local model with GUT gauge group. Apart from demonstrating the CFT techniques developed in the previous section, our primary aim is twofold: First to show that rigid cycles meeting the strong requirements for the generation of 2-point couplings exist even on toroidal backgrounds; and second to demonstrate that the resulting E2-instanton effects do have the potential to yield Majorana mass terms for the right-handed neutrinos within the range $10^{8}-10^{15} \mathrm{GeV}$.

\section{A. Background on the $T^{6} / \mathbb{Z}_{2} \times \mathbb{Z}_{2}^{\prime}$ orientifold}

Consider the orientifold $T^{6} / \mathbb{Z}_{2} \times \mathbb{Z}_{2}^{\prime}$ with Hodge numbers $\left(h_{11}, h_{12}\right)=(3,51)$. We stick to the notation of [17], to which we refer for details of the geometry and the con- struction of rigid cycles. The orbifold group is generated by $\theta$ and $\theta^{\prime}$ acting as reflection in the first and last two tori, respectively.

This background exhibits two types of factorizable special Lagrangian three-cycles. The first class is given by the usual nonrigid bulk cycles

$$
\Pi_{a}^{B}=4 \bigotimes_{I=1}^{3}\left(n_{a}^{I}\left[a^{I}\right]+\tilde{m}_{a}^{I}\left[b^{I}\right]\right),
$$

defined in terms of the fundamental one-cycles $\left[a^{I}\right],\left[b^{I}\right]$ of the $I$ th $T^{2}$ and the corresponding wrapping numbers $n_{a}^{I}$ and $\tilde{m}_{a}^{I}=m_{a}^{I}+\beta^{I} n_{a}^{I}$. Here $\beta^{I}=0,1 / 2$ for rectangular and tilted tori, respectively.

In addition there exist so-called $g$-twisted three-cycles

$$
\Pi_{i j}^{g}=n^{I_{g}}\left[\alpha_{i j, n}^{g}\right]+\tilde{m}^{I_{g}}\left[\alpha_{i j, m}^{g}\right],
$$

where $i, j \in\{1,2,3,4\} \times\{1,2,3,4\}$ labels one of the 16 blown-up fixed points of the orbifold element $g=$ $\theta, \theta^{\prime}, \theta \theta^{\prime} \in \mathbb{Z}_{2} \times \mathbb{Z}_{2}^{\prime}$. The cycles $\left[\alpha_{i j, n}^{g}\right]\left(\left[\alpha_{i j, m}^{g}\right]\right)$ can be understood as twice the product of the corresponding $\mathbb{P}_{1}$ and the one-cycle $[a]^{I_{g}}\left([b]^{I_{g}}\right)$ in the $I_{g}$ th $T^{2}$ invariant under $g$. Here $I_{g}=3,1,2$ for $g=\theta, \theta^{\prime}, \theta \theta^{\prime}$, respectively.

These twisted cycles are the building blocks for certain fractional cycles $\Pi^{F}$ charged under all three twisted sectors. They are rigid and will serve as candidates for E2-branes contributing to the superpotential. The general expression for $\Pi^{F}$ is given by

$$
\begin{aligned}
\Pi^{F}= & \frac{1}{4} \Pi^{B}+\frac{1}{4}\left(\sum_{i, j \in S_{\theta}} \epsilon_{i j}^{\theta} \Pi_{i j}^{\theta}\right)+\frac{1}{4}\left(\sum_{j, k \in S_{\theta^{\prime}}} \epsilon_{j k}^{\theta^{\prime}} \Pi_{j k}^{\theta^{\prime}}\right) \\
& +\frac{1}{4}\left(\sum_{i, k \in S_{\theta \theta^{\prime}}} \epsilon_{i k}^{\theta \theta^{\prime}} \Pi_{i k}^{\theta \theta^{\prime}}\right) .
\end{aligned}
$$

The sets $S_{g}$ denote the four different fixed points in the $g$-twisted sector compatible with the bulk wrapping numbers and the concrete position of the brane, as detailed in [17]. A given set of bulk wrapping numbers allows for a choice of $2 \times 2 \times 2=8$ inequivalent positions of the fractional brane. Each of these branes is further specified by the signs $\epsilon_{i j}^{g}$, corresponding to the orientation with which the various $\mathbb{P}_{1}$ are wrapped in the twisted sector. They are subject to various consistency conditions [17] such that for each choice of position of the fractional brane, there are only 8 inequivalent choices of $\epsilon_{i j}^{g}$.

The orientifold action $\Omega \mathcal{R}$ on the untwisted cycles follows from

$$
\Omega \mathcal{R}:\left[a_{I}\right] \rightarrow\left[a_{I}\right] \quad \Omega \mathcal{R}:\left[b_{I}\right] \rightarrow-\left[b_{I}\right],
$$

whereas the twisted cycles transform as

$$
\begin{aligned}
& \Omega \mathcal{R}: \alpha_{i j, n}^{g} \rightarrow-\eta_{\Omega \mathcal{R}} \eta_{\Omega \mathcal{R} g} \alpha_{\mathcal{R}(i) \mathcal{R}(j), n}^{g}, \\
& \Omega \mathcal{R}: \alpha_{i j, m}^{g} \rightarrow \eta_{\Omega \mathcal{R}} \eta_{\Omega \mathcal{R} g} \alpha_{\mathcal{R}(i) \mathcal{R}(j), m}^{g} .
\end{aligned}
$$

Here the reflection $\mathcal{R}$ leaves all fixed points of an untilted 
two-torus invariant and acts on the fixed points in a tilted two-torus as

$$
\mathcal{R}(1)=1 \quad \mathcal{R}(2)=2 \quad \mathcal{R}(3)=4 \quad \mathcal{R}(4)=3 .
$$

The signs $\eta_{\Omega \mathcal{R} g}= \pm 1$ defining the orientifold action are subject to the constraint

$$
\eta_{\Omega \mathcal{R}} \eta_{\Omega \mathcal{R} \theta} \eta_{\Omega \mathcal{R} \theta^{\prime}} \eta_{\Omega \mathcal{R} \theta \theta^{\prime}}=-1 .
$$

In our subsequent example we choose for simplicity all tori to be untilted and

$$
\eta_{\Omega \mathcal{R}}=\eta_{\Omega \mathcal{R} \theta}=\eta_{\Omega \mathcal{R} \theta \theta^{\prime}}=-\eta_{\Omega \mathcal{R} \theta^{\prime}}=1 .
$$

In this case, the orientifold image of the cycle $\Pi^{F}$ in Eq. (43) is given by $\Pi^{\prime F}$,

$$
\begin{aligned}
\Pi^{\prime F}= & \frac{1}{4} \hat{\Pi}^{B}-\frac{1}{4}\left(\sum_{i, j \in S_{\theta}} \epsilon_{i j}^{\theta} \hat{\Pi}_{i j}^{\theta}\right)+\frac{1}{4}\left(\sum_{j, k \in S_{\theta^{\prime}}} \epsilon_{j k}^{\theta^{\prime}} \hat{\Pi}_{j k}^{\theta^{\prime}}\right) \\
& -\frac{1}{4}\left(\sum_{i, k \in S_{\theta \theta^{\prime}}} \epsilon_{i k}^{\theta \theta^{\prime}} \hat{\Pi}_{i k}^{\theta \theta^{\prime}}\right),
\end{aligned}
$$

where the ${ }^{\wedge}$ denotes the substitution $m^{I} \rightarrow-m^{I}$ (see also [18]).

The fixed point locus sets expressed in terms of the toroidal cycles take the form

$$
\begin{aligned}
\pi_{O 6}= & 2\left[a_{1}\right]\left[a_{2}\right]\left[a_{3}\right]-2\left[b_{1}\right]\left[b_{2}\right]\left[a_{3}\right]+2\left[a_{1}\right]\left[b_{2}\right]\left[b_{3}\right] \\
& -2\left[b_{1}\right]\left[a_{2}\right]\left[b_{3}\right] .
\end{aligned}
$$

We also recall the topological intersection number $I_{a b}$ of two bulk branes $\Pi_{a}^{B}$ and $\Pi_{b}^{B}$,

$$
I_{a b}=4 \prod_{i=1}^{3}\left(n_{a}^{i} m_{b}^{i}-n_{b}^{i} m_{a}^{i}\right)
$$

Since our conventions are such that a stack of $N_{a}$ coincident branes away from the orientifold carries gauge group $U\left(N_{a} / 2\right)$ upon taking the $\mathbb{Z}_{2}$ projection on the Chan-Paton factors into account, the quantity (50) counts the number of chiral multiplets in the bifundamental of the gauge group $\left(\overline{\mathbf{N}}_{\mathrm{a}} / \mathbf{2}, \mathbf{N}_{\mathbf{b}} / \mathbf{2}\right)$ living at the intersection of two stacks of $N_{a}$ and $N_{b}$ bulk cycles $a$ and $b$, respectively. The number of chiral multiplets transforming as antisymmetric and symmetric representations under $U\left(N_{a} / 2\right)$ is

$$
I^{\mathrm{anti}}=\frac{1}{2}\left(I_{a^{\prime} a}+I_{O 6 a}\right), \quad I^{\mathrm{sym}}=\frac{1}{2}\left(I_{a^{\prime} a}-I_{O 6 a}\right),
$$

where

$$
\begin{aligned}
I_{a a^{\prime}} & =-32 n_{a}^{1} m_{a}^{1} n_{a}^{2} m_{a}^{2} n_{a}^{3} m_{a}^{3}, \\
I_{O 6 a} & =8 m_{a}^{1} m_{a}^{2} m_{a}^{3}-8 n_{a}^{1} n_{a}^{2} m_{a}^{3}+8 m_{a}^{1} n_{a}^{2} n_{a}^{3}-8 n_{a}^{1} m_{a}^{2} n_{a}^{3} .
\end{aligned}
$$

In our applications the E2-instanton will wrap a rigid cycle $\Xi$. Its intersection with a bulk brane $\Pi_{a}^{B}$ and its image $\left(\Pi_{a}^{B}\right)^{\prime}$ is independent of the twisted charge of $\Xi$,

$$
\begin{gathered}
I_{\Xi a}=\prod_{i=1}^{3}\left(n_{\Xi}^{i} m_{a}^{i}-n_{a}^{i} m_{\Xi}^{i}\right), \\
I_{\Xi a^{\prime}}=-\prod_{i=1}^{3}\left(n_{\Xi}^{i} m_{a}^{i}+n_{a}^{i} m_{\Xi}^{i}\right) .
\end{gathered}
$$

\section{B. Wrapping numbers and spectrum of a local brane setup}

We proceed with the construction of a local $S U(5)$ GUTlike model. In this approach, ${ }^{13}$ the standard model arises from a stack of 10 coincident $D 6$-branes carrying gauge group $U(5)=S U(5) \times U(1)$, where the Abelian part is massive due to the Green-Schwarz mechanism. For simplicity, we choose the GUT stack to be given by nonrigid bulk branes so that the GUT group can be broken down to the standard model gauge group by invoking branesplitting, i.e., by giving suitable vacuum expectation values to the GUT Higgs fields in the adjoint of $S U(5)$. Righthanded neutrinos are localized at the intersection of two more stacks $a$ and $b$ of D-branes such that they are indeed singlets under the GUT $S U(5)$. We choose $a$ and $b$ to be likewise given by bulk cycles. The actual "standard model" spectrum arises, upon GUT breaking, from 4 chiral generations in the $\mathbf{1 0}$ of $S U(5)$ as well as chiral multiplets transforming as $\overline{\mathbf{5}}$ localized at the intersection of $c$ and $a$. The electroweak Higgs field candidates $\mathbf{5}_{H}$ arise from the intersection between stack $c$ and $b$. In Table I we display the wrapping numbers of the stacks $a, b$, and $c$ of D-branes in a particular realization of the described local setup. This table also contains the intersection numbers of the stacks and their image stacks with the E2-instanton to be defined later in Eq. (58). Table II gives the multiplicities of the "standard model" spectrum. In addition, there is chiral exotic matter which we do not make explicit.

Note that the charges $\left(-1_{a}, 1_{b}\right)$ of $N_{R}^{c}$ under the global symmetries $U(1)_{a}$ and $U(1)_{b}$ indeed forbid perturbative Majorana masses. We therefore seek to generate such terms nonperturbatively. In order for the potential instanton induced Majorana mass terms to yield, via the standard seesaw mechanism, hierarchically small masses for the neutrino mass eigenstates, the model has to allow for perturbatively generated Dirac neutrino masses.

This feature is indeed realized, as can be seen from the concrete intersection pattern in Table II. The Dirac mass terms are encoded schematically in the coupling

$$
H L_{L} N_{R}^{c} \in \mathbf{5}_{H} \overline{\mathbf{5}} \mathbf{1} .
$$

The magnitude of Dirac mass terms depends on the magnitude of the above Yukawa couplings and the vacuum expectation value of the standard model Higgs fields $H$. Yukawa couplings are determined by a tree-level disk amplitude calculation $[28,30]$. They are in general expo-

\footnotetext{
${ }^{13}$ See, e.g., [31,32] for global constructions of a similar type.
} 
TABLE I. Wrapping numbers of the local setup.

\begin{tabular}{lrcr}
\hline \hline Stack & $N$ & $\left(n^{1}, m^{1}\right) \times\left(n^{2}, m^{2}\right) \times\left(n^{3}, \tilde{m}^{3}\right)$ & $I_{E 2 x}$ \\
\hline$E_{2}$ & 1 & $(1,0) \times(0,1) \times(0,-1)$ & $\cdots$ \\
$a$ & 2 & $(1,2) \times(1,1) \times(-1,-1)$ & 2 \\
$b$ & 2 & $(-3,-2) \times(1,-1) \times(-1,0)$ & -2 \\
$c$ & 10 & $(1,0) \times(-1,2) \times(-2,-3)$ & 0 \\
\hline \hline
\end{tabular}

TABLE II. Matter spectrum of the local setup.

\begin{tabular}{lrcc}
\hline \hline Sector & \multicolumn{1}{c}{$I_{x y}$} & Representation & Matter \\
\hline$\left(c, c^{\prime}\right)$ & 4 & Antisymmetric & $\mathbf{1 0}$ \\
$(c, a)$ & 24 & $(\bar{c}, a)$ & $\overline{\mathbf{5}}$ \\
$(c, b)$ & -24 & $(c, \bar{b})$ & $\mathbf{5}_{H}$ \\
$(a, b)$ & 32 & $(\bar{a}, b)$ & $N_{R}^{c}$ \\
\hline \hline
\end{tabular}

nentially suppressed by the area of the leading triangle formed by the intersecting branes and thus depend on the Kähler and open string moduli of the background in the way found in $[28,30]$. For a toroidal-type setup, constraints on the four-dimensional gauge couplings and Planck length $\ell_{\text {Planck }}$ typically constrain $\ell_{s} \sim \ell_{\text {Planck }}$ and thus the terms in the leading exponents typically cannot be larger than $\mathcal{O}(10)$. For an early concrete analysis of these suppression terms for the first globally consistent three-family supersymmetric standardlike model $[33,34]$, see [35]. The analysis of Dirac mass terms can be repeated in a straightforward way for our setup by parallel splitting of GUT $S U$ (5) branes into three SM branes and analyzing the associated Yukawa couplings for quarks and leptons. In general Dirac neutrino masses will follow the mass pattern of charged leptons and quarks, and are thus in the same mass range.

In order for the model to be supersymmetric each stack of branes has to satisfy the two conditions [17]

$$
m_{x}^{1} m_{x}^{2} m_{x}^{3}-\sum_{I \neq J \neq K} \frac{n_{x}^{I} n_{x}^{J} m_{x}^{K}}{U^{I} U^{J}}=0
$$

and

$$
n_{x}^{1} n_{x}^{2} n_{x}^{3}-\sum_{I \neq J \neq K} m_{x}^{I} m_{x}^{J} n_{x}^{K} U^{I} U^{J}>0,
$$

where $U^{I}$ denotes the complex structure modulus $U^{I}=$ $R_{Y}^{I} / R_{X}^{I}$ of the $I$ th torus with radii $R_{X}^{I}, R_{Y}^{I}$. The brane setup satisfies the equations above for the following choice of complex structure moduli $U^{I}$,

$$
U^{1}=\sqrt{3}, \quad U^{2}=\frac{2}{\sqrt{3}}, \quad U^{3}=\frac{8}{3 \sqrt{3}} .
$$

We stress once more that the brane configuration of Table I as such does not satisfy all of the tadpole cancellation conditions ensuring global consistency and therefore only represents a local model. In particular, its spectrum is not anomaly free.

\section{E2-instanton}

We are now in a position to analyze the E2-instanton sector of the local model defined in the previous section. We are particularly interested in fermion bilinears of type (36) for the right-handed neutrinos. As described in detail, they are due to $E 2$-instantons wrapping a rigid supersymmetric cycle $\Xi$ subject to (21) such that the $\bar{\theta}_{\dot{\alpha}}$ modes are projected out [21] and which do not give rise to zero modes in the $\Xi-\Xi^{\prime}$ sector. Our analysis therefore consists in two steps: First classify the rigid cycles $\Xi$ with no $\Xi-\Xi^{\prime}$ modes and then distinguish them according to their charged zero modes structure. As it will turn out, in our setup the first step automatically guarantees absence of the $\bar{\theta}_{\dot{\alpha}}$ modes as well.

The only type of sLags under technical control corresponds to the class of factorizable three-cycles described in Sec. III A. Even though a complete analysis of the instanton sector should take into account all possible sLags our analysis is therefore forced to content itself with this special class. A closer look reveals that the constraint $\Xi \cap$ $\Xi^{\prime}=0$ is extremely restrictive and can be met (at best) by two different types of rigid cycles. The first corresponds to $\Xi$ and its orientifold image $\Xi^{\prime}$ being parallel, but separated in at least one of the three tori. In that case the vectorlike fermionic zero modes in the $\Xi-\Xi^{\prime}$ sector carry a mass proportional to the distance between $\Xi$ and $\Xi^{\prime}$. Alternatively, one can consider those rigid cycles with the property $\Xi=\Xi^{\prime}$. For such invariant cycles, the massless modes in the $E 2-E 2^{\prime}$ sector are identical to the geometric moduli of the cycle. Rigidity then guarantees the absence of open string moduli and $E 2-E 2^{\prime}$ zero modes at the same time.

As is immediately clear, the explicit form of the orientifold action on the fixed points in the $\mathbb{Z}_{2} \times \mathbb{Z}_{2}^{\prime}$ background at hand excludes the first type of cycles. Potential candidate cycles for the second class have to lie on top of one of the four orientifold planes to ensure that the bulk part is indeed mapped to itself under $\Omega \mathcal{R}$. In addition, we have to take into account the nontrivial orientifold action on the $g$-twisted sector encoded in (45). Depending on the choice of $\eta_{\Omega \mathcal{R} g}$, a certain combination of twisted charges of $\Xi$ may also be $\Omega$ invariant such that $\Xi=\Xi^{\prime}$. With our given choice (48) for $\eta_{\Omega \mathcal{R}_{g}}$, only those rigid cycles parallel to the $x^{1}, y^{2}$, and $y^{3}$ axis have a chance to be invariant. This can be seen, e.g., from the fact that the $\alpha_{i j, m}^{g}$ are invariant only for $g=\theta$ and $g=\theta \theta^{\prime}$, cf. Eq. (45). Because of the additional minus sign in the orientifold projection resulting from the external Dirichlet-Dirichlet boundary conditions, the nondynamical gauge group on a stack of $N$ such invariant instantons is $S O(N) .{ }^{14}$ In particular, this means that we can wrap a single instanton on this invariant cycle

\footnotetext{
${ }^{14}$ Recall that for D6-branes wrapping invariant cycles, the gauge group was determined in [17] to be $\operatorname{Sp}(2 N)$.
} 
and that the orientifold projection removes the unwanted $\bar{\theta}_{\dot{\alpha}}$ modes from the $E 2-E 2$ sector.

To completely specify a cycle of this type we have to choose the explicit values for the bulk wrapping numbers, the actual position of the brane and thus the fixed points $S_{g}$ to be wrapped in the twisted sector, and finally the $\epsilon^{g}$ signs.

We start with the bulk wrapping numbers.

From (21) we need intersection numbers $I_{E 2 a}=2$ and $I_{E 2 b}=-2$ in order for the instanton to exhibit Abelian charges $Q_{a}=2$ and $Q_{b}=-2$. Since $E 2=E 2^{\prime}$, the zero modes in the $E 2-a$ and $a^{\prime}-E 2$ are identified and do not therefore count as independent. This uniquely determines the bulk wrapping numbers of the fractional cycle to

$$
\Pi_{\Xi}^{B}:[(1,0)(0,1)(0,-1)] .
$$

We note in passing that by this analysis there exist no $E 2$-instantons leading to dangerous open string tadpoles of the form $\Phi e^{-S_{\text {inst }}}$ for matter between the stacks $a, b$, or $c$. For perturbatively well-defined string vacua such tadpoles would spoil stability at the quantum level.

Given the bulk wrapping numbers (57), we have the following options for the twisted sector: The fractional brane can run through the fixed points $(1,3)$ or $(2,4)$ in the first torus and through $(1,2)$ or $(3,4)$ in the second and third torus (see Fig. 2).

Thus, we have 8 different positions for the invariant cycle, together with the mentioned 8 inequivalent sign choices $\epsilon^{g}$ for each position. One example of these 64 different cycles takes the form

$$
\begin{aligned}
\Pi_{\Xi}= & \frac{1}{4} \Pi_{\Xi}^{B}-\frac{1}{4} \sum_{i, j \epsilon(13) \times(12)} \alpha_{i j, m}^{\theta}+\frac{1}{4} \sum_{j, k \in(12) \times(12)} \alpha_{j k, n}^{\theta^{\prime}} \\
& +\frac{1}{4} \sum_{i, k \in(13) \times(12)} \alpha_{i k, m}^{\theta \theta^{\prime}} .
\end{aligned}
$$

It corresponds to an $E 2$ passing through the origin in each of the three tori and the choice $\epsilon_{i j}^{g}=1$ in all sectors, as depicted in Fig. 2. The remaining 63 instanton cycles are obvious modifications of this one. One may convince oneself that the choice (48) indeed yields $\Xi=\Xi^{\prime}$, thus qualifying $\Xi$ as an $E 2$-instanton cycle relevant for the superpotential.

In the sequel, when analyzing the single E2-instanton sector relevant for the Majorana mass terms, we have to consider each of these inequivalent choices of the twisted sector. The final result for the nonperturbative coupling will be the sum of the contribution from each sector. Our explicit computation will be for instanton (58) and we will discuss the remaining contributions at the end of Sec. III D.

It is crucial for the generation of fermion bilinears that there exist no chiral zero modes from strings stretching between the $E 2$-instanton and the stack $c$ since $I_{\Xi c}=0$. However, since $\Xi$ and $c$ share the same bulk wrapping numbers in the first torus, there exist vectorlike pairs at the intersection of $\Xi$ and $c$ in the second and third torus with mass proportional to twice the distance between $\Xi$ and $c$ in the first torus. In order to avoid massless vectorlike pairs, we have to assume that the latter stack is separated from the instanton in the second torus by a nonzero distance. In the absence of effects stabilizing the open string moduli, we can freely move along the corresponding flat direction in moduli space.

To summarize, the zero-mode structure meets the required constraints to give rise to Majorana mass terms for the right-handed neutrinos $\nu_{R}^{c}$ sitting in the superfields $N_{R}^{c}$ at the intersection of branes $(a, b)$ carrying Abelian charge $\left(-1_{a}, 1_{b}\right)$ (see Table II). One may check that $U(1)_{a}$ and $U(1)_{b}$ are indeed both broken as a gauge symmetry since the corresponding vector potentials acquire a Stückelbergtype mass. Recall that this is the conditio sine qua non for the instanton to offset the Abelian charge violation of the open string operator in the nonperturbative coupling.

\section{Computation of the Majorana masses}

We finally apply the results of Sec. IIC and obtain the neutrino Majorana mass terms for the local model described in Sec. III B and III C by evaluating the two-point
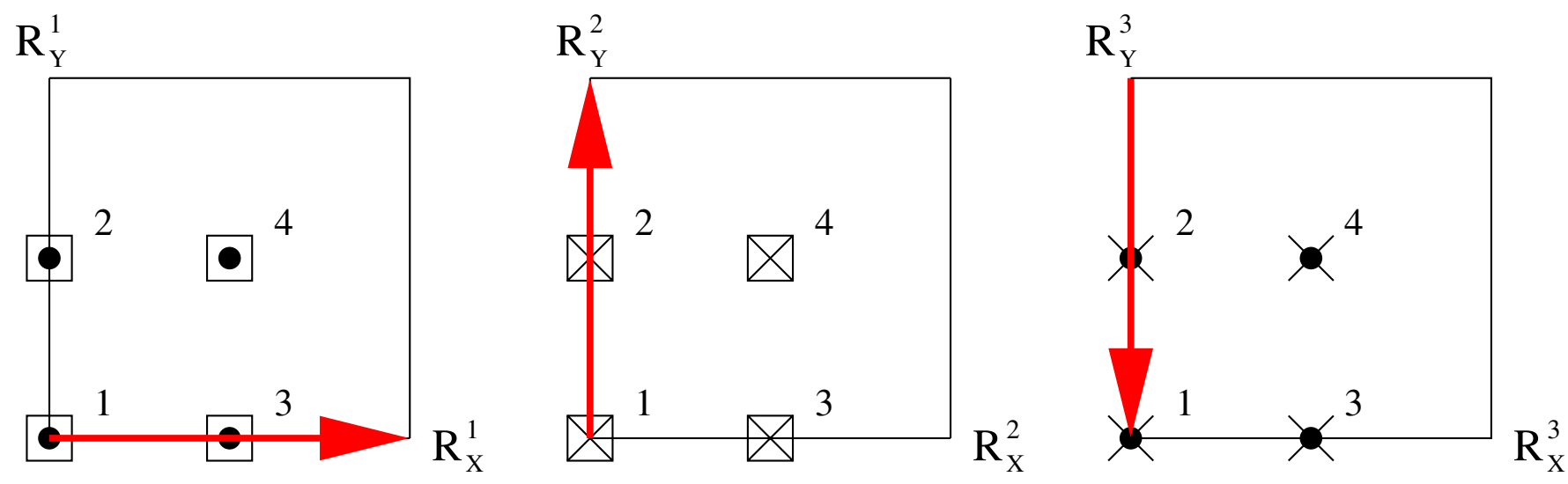

FIG. 2 (color online). $\quad T^{6} / \mathbb{Z}_{2} \times \mathbb{Z}_{2}^{\prime}$ with $\beta^{1}=\beta^{2}=\beta^{3}=0$. 
correlator

$$
\begin{aligned}
\left\langle\nu^{A} \nu^{B}\right\rangle_{E 2}= & -\frac{1}{2 !} \frac{V_{E 2}}{16} \frac{g_{s}}{2 \pi} \int d^{4} x_{E} \int d^{2} \theta \int d^{2} \lambda_{a} \\
& \times \int d^{2} \bar{\lambda}_{b} e^{-S_{\text {inst }}} e^{Z^{\prime}} \\
& \times \sum_{i, j, k, l}\left\langle V_{\Theta^{\alpha}}^{-(1 / 2)} V_{\bar{\lambda}_{b}^{k}}^{-(1 / 2)} V_{\nu_{\alpha}^{A}}^{-(1 / 2)} V_{\lambda_{a}^{i}}^{-(1 / 2)}\right\rangle \\
& \times\left\langle V_{\Theta^{\beta}}^{-(1 / 2)} V_{\bar{\lambda}_{b}^{I}}^{-(1 / 2)} V_{\nu_{\beta}^{B}}^{-(1 / 2)} V_{\lambda_{a}^{j}}^{-(1 / 2)}\right\rangle .
\end{aligned}
$$

For the concrete intersection angles corresponding to the setup in III B,

$$
\begin{aligned}
& \theta_{a b}^{1}=0.86, \quad \theta_{a b}^{2}=-0.54, \quad \theta_{a b}^{3}=-0.32, \\
& \theta_{E 2 a}^{1}=0.41, \quad \theta_{E 2 a}^{2}=-0.23, \quad \theta_{E 2 a}^{3}=-0.18, \\
& \theta_{E 2 b}^{1}=-0.73, \quad \theta_{E 2 b}^{2}=-0.77, \quad \theta_{E 2 b}^{3}=-0.50 \text {, }
\end{aligned}
$$

the vertex operators read

$$
\begin{aligned}
V_{\nu}= & \ell_{s}^{3 / 2} \Omega_{b a} \nu_{\alpha} S^{\alpha}(z) \sigma_{1-\theta_{a b}^{1}}(z) e^{-i\left(\theta_{a b}^{1}-1 / 2\right) H_{1}(z)} \\
& \times \prod_{I=2}^{3} \sigma_{-\theta_{a b}^{I}}(z) e^{-i\left(\theta_{a b}^{I}+1 / 2\right) H_{I}(z)} e^{i k_{\mu} X^{\mu}(z)} e^{-\varphi(z) / 2}, \\
V_{\lambda_{a}}= & \Omega_{a E 2} \lambda_{a} \Sigma(z) \sigma_{1-\theta_{E 2 a}^{1}}(z) e^{-i\left(\theta_{E 2 a}^{1}-1 / 2\right) H_{1}(z)} \\
& \times \prod_{I=2}^{3} \sigma_{-\theta_{E 2 a}^{I}}(z) e^{-i\left(\theta_{E 2 a}^{I}+1 / 2\right) H_{I}(z)} e^{-\varphi(z) / 2}, \\
V_{\bar{\lambda}_{b}}= & \Omega_{E 2 b} \bar{\lambda}_{b} \Sigma(z) \prod_{I=1}^{3} \sigma_{1+\theta_{E 2 b}^{I}}(z) e^{i\left(\theta_{E 2 b}^{I}+1 / 2\right) H_{I}(z)} e^{-\varphi(z) / 2} .
\end{aligned}
$$

It follows that the angle dependence of the disk amplitude

$$
\begin{gathered}
\left\langle V_{\Theta^{\alpha}}^{-(1 / 2)} V_{\bar{\lambda}_{b}^{k}}^{-(1 / 2)} V_{\nu_{\alpha}^{A}}^{-(1 / 2)} V_{\lambda_{a}^{i}}^{-(1 / 2)}\right\rangle=\frac{2 \pi}{g_{s}} \ell_{s}^{3 / 2} C_{i k}^{A} e^{-i k_{\mu}^{A} x_{0}^{\mu}} \\
\left(\theta^{\alpha} \bar{\lambda}_{b}^{k} \nu_{\alpha}^{A} \lambda_{a}^{i}\right)
\end{gathered}
$$

is given by

$$
\begin{aligned}
C_{i k}^{A}= & \pi\left[4 \pi \Gamma_{1-\theta_{a b}^{1}, 1-\theta_{E 2 a}^{1}, 1+\theta_{E 2 b}^{1}} \prod_{I=2}^{3} 4 \pi \Gamma_{-\theta_{a b}^{I},-\theta_{E 2 a}^{I}, 1+\theta_{E 2 b}^{I}}\right]^{1 / 4} \\
& \times \sum_{m_{j}} e^{-\mathcal{A}_{i k}^{A}\left(m_{j}\right)}
\end{aligned}
$$

for index combinations with nonvanishing diagrams.
Before turning to this question, we first investigate the instanton suppression factor

$$
e^{-S_{\text {inst }}}=e^{-\left(2 \pi / \ell_{s}^{3} g_{s}\right) \operatorname{Vol}_{E 2}}=e^{-\left(2 \pi / \alpha_{\mathrm{GUT}}\right)\left(\operatorname{Vol}_{E 2} / \operatorname{Vol}_{\Pi_{c}}\right)},
$$

where the last equation uses the standard relation (see, e.g., [36])

$$
\alpha_{\mathrm{GUT}}=g_{s} \frac{\mathrm{Vol}_{\Pi_{c}}}{\ell_{s}^{3}}
$$

for $\alpha_{\mathrm{GUT}}$ in terms of the volume of the GUT stack $c$. Given the geometric data of our concrete string vacuum, the ratio $\mathrm{Vol}_{E 2} / \mathrm{Vol}_{\Pi_{a}}$ can easily be computed and is determined entirely by the wrapping numbers in Table I and the complex structure moduli (56),

$$
\frac{\mathrm{Vol}_{E 2}}{\operatorname{Vol}_{\Pi_{c}}}=\left(\prod_{I} \frac{\left(n_{E 2}^{I}\right)^{2}+\left(\tilde{m}_{E 2}^{I}\right)^{2} U_{I}^{2}}{\left(n_{c}^{I}\right)^{2}+\left(\tilde{m}_{c}^{I}\right)^{2} U_{I}^{2}}\right)^{1 / 2}=\frac{8}{57} .
$$

As discussed in Sec. II D, for $C_{i k}^{A}$ to be nonvanishing in each torus the modes $\lambda_{a}^{i}, \bar{\lambda}_{b}^{k}$, and $\nu^{A}$ have to form a triangle. Let us analyze this nontrivial constraint for our setup. Figure 3 displays the intersection in the first torus.

One can easily read off that the combinations of $\lambda_{a}^{i}, \bar{\lambda}_{b}^{k}$, and $\nu^{A}$ with triangles in the first torus have the same structure as in the example in section II D.

The remaining two tori are depicted in Fig. 4. While $a$ and $b$ intersect twice in the second torus there is only one intersection in the third one. Most importantly, the repli-

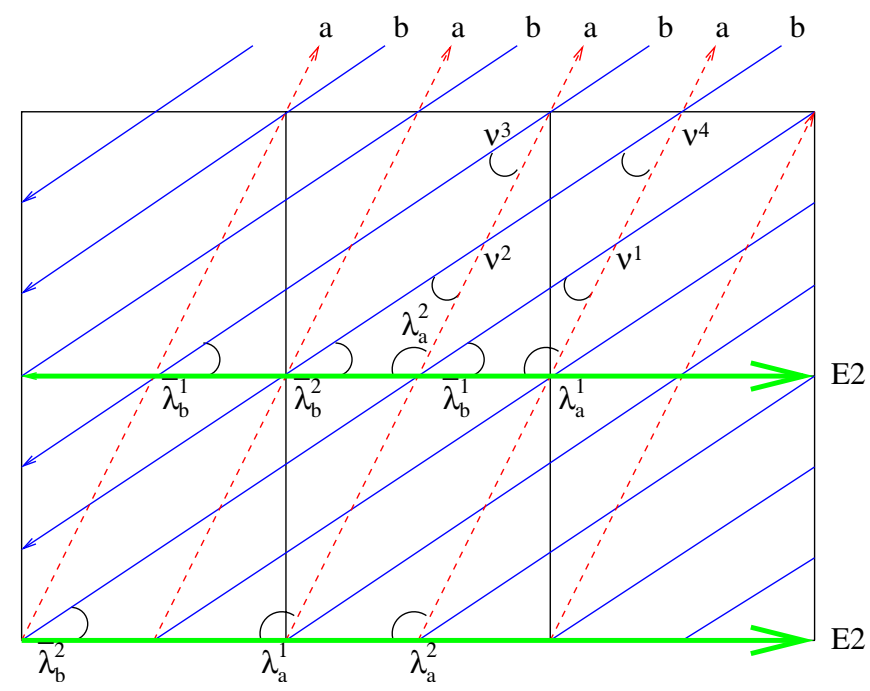

FIG. 3 (color online). Intersection pattern in first torus. 
a)

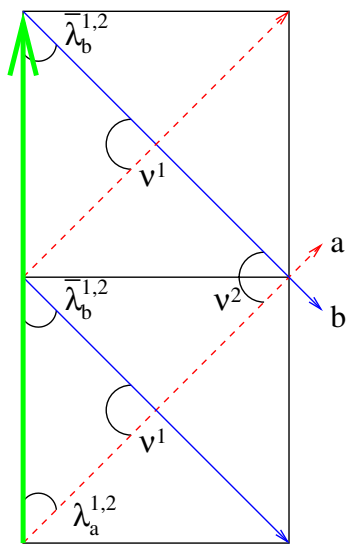

b)

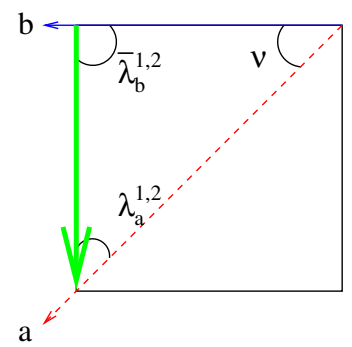

FIG. 4 (color online). Intersection pattern in second (a) and third (b) torus.

cation of $\lambda_{a}$ and $\bar{\lambda}_{b}$ modes is entirely due to multiple intersections in the first torus.

The complete location of a neutrino $\nu^{i, j}$ is described by two upper indices $i$ and $j$, where $i$ denotes the position in the first torus while $j$ gives the location in the second. ${ }^{15}$ Ignoring all higher world sheet instanton effects we obtain

$$
\left\langle\nu^{A} \nu^{B}\right\rangle_{E 2}=\frac{2 \pi V_{E 2}}{g_{s}} \vec{v}^{T} \mathcal{M} \vec{v}(2 \pi)^{4} \delta^{4}\left(k^{A}+k^{B}\right) .
$$

Here $\vec{v}$ is defined as

$$
\vec{v}^{T}=\left(\nu^{1,1}, \nu^{2,1}, \nu^{3,1}, \nu^{4,1}, \nu^{1,2}, \nu^{2,2}, \nu^{3,2}, \nu^{4,2}\right)
$$

and the $8 \times 8$ matrix $\mathcal{M}$ takes the form

$$
\mathcal{M}=x M_{s} e^{-\left(16 \pi / 57 \alpha_{\mathrm{GUT}}\right)}\left(\begin{array}{cccc}
A & 0 & B & 0 \\
0 & C & 0 & D \\
B & 0 & E & 0 \\
0 & D & 0 & F
\end{array}\right),
$$

where $x$ is given by

$$
x=\frac{\pi^{2}}{16}\left[4 \pi \Gamma_{1-\theta_{a b}^{1}, 1-\theta_{E 2 a}^{1}, 1+\theta_{E 2 b}^{1}} \prod_{I=2}^{3} 4 \pi \Gamma_{-\theta_{a b}^{I},-\theta_{E 2 a}^{I}, 1+\theta_{E 2 b}^{I}}\right]^{1 / 2} e^{Z^{\prime}} .
$$

At "tree level," i.e., ignoring the corrections due to the one-loop determinant $e^{Z}$, the numerical factor is approximately $x \approx 0.87$. The building blocks of $\mathcal{M}$ in (69) take a similar form as in the simpler example in Sec. IID,

$$
\begin{gathered}
A=\left(\begin{array}{cc}
e^{-(\alpha+\beta+2 \kappa+2 \tau)} & \frac{1}{2}\left(e^{-2(\alpha+\kappa+\tau)}+e^{-2(\beta+\kappa+\tau)}\right) \\
\frac{1}{2}\left(e^{-2(\alpha+\kappa+\tau)}+e^{-2(\beta+\kappa+\tau)}\right) & e^{-(\alpha+\beta+2 \kappa+2 \tau)}
\end{array}\right) \\
B=\left(\begin{array}{cc}
e^{-(\alpha+\beta+\kappa+\mu+2 \tau)} & \frac{1}{2}\left(e^{-(2 \alpha+\kappa+\mu+2 \tau)}+e^{-(2 \beta+\kappa+\mu+2 \tau)}\right) \\
\frac{1}{2}\left(e^{-(2 \alpha+\kappa+\mu+2 \tau)}+e^{-(2 \beta+\kappa+\mu+2 \tau)}\right) & e^{-(\alpha+\beta+\kappa+\mu+2 \tau)}
\end{array}\right),
\end{gathered}
$$

where $\alpha, \beta, \gamma$, and $\delta$ are defined in (39), $\kappa(\mu)$ denotes the area of the triangle spanned by $\nu^{i, 1}\left(\nu^{i, 2}\right), \lambda_{a}$, and $\bar{\lambda}_{b}$ in the second torus, whereas $\tau$ is the area in the third torus. The other 4 building blocks can be easily obtained in the following manner. Replacing $\alpha$ and $\beta$ in $A(B)$ by $\gamma$ and $\delta$ yields $C(D)$, replacing in addition also $\kappa$ by $\mu$ one obtains $F$. In order to get $E$ we just substitute in $A \kappa$ by $\mu$.

The suppression due to world sheet instantons depends crucially on the open string moduli. However, since for a toroidal-type setup the four-dimensional Planck length $\ell_{\text {Planck }}$ is constrained to be of the same order of magnitude as $\ell_{s}$, the arguments of the leading exponents typically range between zero and order one, and thus these suppression factors are not excessive. In this case, also the factor $V_{E 2}$ in (67) is of order 1. In addition, for particular choices of open string moduli the area of triangles vanishes and there is no suppression at all, e.g., for the instanton passing

\footnotetext{
${ }^{15}$ Recall that $a$ and $b$ are bulk branes so that each intersection point gives rise to 4 right-handed neutrinos $\nu_{R}^{c} \equiv \nu$. This yields the overall $4 \times 8=32$ of them. We leave the additional factor of 4 implicit.
}

through the origin in each torus and the choice of moduli as in Fig. 3 and 4 there is no suppression for the coupling $\nu^{3,2} \nu^{4,2}$.

As discussed, the above coupling is the contribution of just 1 out of $8 \times 8$ rigid factorizable sLags with the required zero-mode structure to yield Majorana mass terms. The first factor is due to the two different positions of the E2-brane per two-torus, corresponding to which of the fixed points it passes through (see Fig. 2). Clearly, each of these 8 choices comes with different areas of the world sheet triangles and therefore relative suppression factors between families. For each geometric position we have to sum in addition over 8 inequivalent choices of signs of the twisted charges $\epsilon_{i j}^{g}$. In our example with all other D6-branes of the local model wrapping bulk cycles, the result for the two-point coupling is independent of these twisted charges. In particular, this is true for the one-loop determinant $e^{Z^{\prime}}$ (17) since the twist part of the $E 2$ boundary state is orthogonal to the boundary states of the bulk D6-branes and the crosscap. It follows that each of the 8 geometrically distinct sectors just contributes with an additional factor of 8 , i.e., the various contributions from the 
factorizable rigid E2-instantons with appropriate zero modes do add up to a nonvanishing result. This is a fortunate result since in principle, one might have feared nontrivial cancellations. Indeed, for heterotic $(0,2)$ models explicit examples of such cancellations are known for special constructions such as (half-)linear sigma models [23], even though they do not correspond to the generic situation. Of course, a complete classification of instanton effects would require control over all special Lagrangian manifolds and seems out of reach even on toridal backgrounds.

For a concrete choice of moduli, it is clear how to perform the sum over instanton configurations in detail. As just discussed, in a crude approximation we can take the suppression due to the areas spanned by the world sheet instantons to be of order one. The summation over the 64 $E 2$-instantons yields an additional factor of $\mathcal{O}(10)$. With $M_{s}=1.2 \times 10^{18} \mathrm{GeV}$ and for $\alpha_{\mathrm{GUT}}$ within the range of $\frac{1}{24}$ and $\frac{1}{20}^{16}$ the factor in (69) takes values in the range of $(0.1-1) \times 10^{11} \mathrm{GeV}$. Therefore for the pattern of neutrino Dirac masses that are in the electroweak range $(0.01-1) \mathrm{GeV}$, the seesaw neutrino masses are in the range $\left(10^{-6}-0.1\right) \mathrm{eV}$.

\section{DISCUSSION}

In this paper, we have continued the analysis of [1] and provided the basic building blocks for determining E2-instanton induced open string superpotential couplings in toroidal type IIA orientifolds. Specifically, we have computed disk diagrams with insertion of one charged matter field and appropriate instanton zero modes. These can then be combined into nonperturbative $M$-point couplings. For the simplest case of fermion bilinears, the exact result is given by (33) and (36). Such terms are of some phenomenological interest since they can represent MSSM $\mu$ terms or Majorana masses for right-handed neutrinos.

Focusing on the latter possibility, we have embedded $E 2$-instanton effects into a local toy model on the $\mathbb{Z}_{2} \times \mathbb{Z}_{2}^{\prime}$ orientifold constructed such that the zero-mode structure of its instanton sector meets all requirements for the genera-

\footnotetext{
${ }^{16}$ Note that the familiar value $\alpha_{\mathrm{GUT}} \simeq 1 / 24$ refers to the exact MSSM spectrum. Given the large amount of exotic matter of our setup we took a range of $\alpha_{\mathrm{GUT}}$ values that is somewhat larger than that of the MSSM.
}

tion of Majorana masses within the phenomenologically allowed window. Together with perturbatively generated Dirac masses, these give rise, via the seesaw mechanism, to hierarchically small neutrino masses. The family mixing pattern among the various Majorana couplings depends crucially on the relative suppression factor governed, as for string tree-level Yukawa couplings, by world sheet instanton effects $[28,30]$. A detailed analysis of the resulting neutrino phenomenology in more realistic models might be of some interest. Since our CFT results are directly applicable also to nonperturbative MSSM $\mu$ terms, the construction of at least local models featuring this effect might also be worthwhile.

As we have described, within the tractable class of rigid factorizable sLags on the $\mathbb{Z}_{2} \times \mathbb{Z}_{2}^{\prime}$ orientifold, the requirement of absence of zero modes in the $E 2-E 2^{\prime}$ sector singles out a small set of candidate cycles for the instanton lying on top of one of the orientifold planes. This is a major challenge for more realistic model building on toroidal backgrounds. The main problem is that, to avoid unacceptable charged zero modes between the instanton and other D6-branes beyond the ones hosting the right-handed neutrinos, these branes have to be parallel to the instanton and separated in one torus. As it turned out in the cases considered, the use of such cycles makes it extremely hard to satisfy all tadpole constraints in a supersymmetric setup. All this comes as no big surprise in view of the simple homology lattice of toroidal backgrounds, and we do not expect these complications to be unsurpassable within the string landscape. In fact, finding M-theory corners naturally incorporating such effects might serve as a guideline in string model building.

\section{ACKNOWLEDGMENTS}

It is our great pleasure to thank Ralph Blumenhagen for important discussions and comments on a draft of this article as well as Luis Ibáñez and Angel Uranga for correspondence on the Goldstone fermions. We also thank Vijay Balasubramanian, Volker Braun, Tamaz Brelidze, Michael Douglas, Paul Langacker, Luca Mazzucato, Tao Liu, Diester Lüst, Mike Schulz, Maximilian SchmidtSommerfeld, Jaemo Park, and Erik Plauschinn for interesting conversations. This research was supported in part by the Department of Energy Grant No. DOE-EY-76-02-3071 and the Fay R. and Eugene L. Langberg Endowed Chair.
[1] R. Blumenhagen, M. Cvetič, and T. Weigand, Nucl. Phys. B771, 113 (2007).

[2] M. Haack, D. Krefl, D. Lüst, A. Van Proeyen, and M. Zagermann, J. High Energy Phys. 01 (2007) 078.
[3] L. E. Ibáñez and A. M. Uranga, J. High Energy Phys. 03 (2007) 052

[4] B. Florea, S. Kachru, J. McGreevy, and N. Saulina, J. High Energy Phys. 05 (2007) 024. 
[5] M. Buican, D. Malyshev, D. R. Morrison, M. Wijnholt, and H. Verlinde, J. High Energy Phys. 01 (2007) 107.

[6] N. Akerblom, R. Blumenhagen, D. Lüst, E. Plauschinn, and M. Schmidt-Sommerfeld, J. High Energy Phys. 04 (2007) 076.

[7] S. A. Abel and M. D. Goodsell, arXiv:hep-th/0612110.

[8] R. Blumenhagen, G. Honecker, and T. Weigand, J. High Energy Phys. 06 (2005) 020.

[9] R. Blumenhagen, G. Honecker, and T. Weigand, J. High Energy Phys. 08 (2005) 009.

[10] R. Blumenhagen, M. Cvetič, P. Langacker, and G. Shiu, Annu. Rev. Nucl. Part. Sci. 55, 71 (2005).

[11] R. Blumenhagen, B. Körs, D. Lüst, and S. Stieberger, Phys. Rep. 445, 1 (2007).

[12] F. Marchesano, Fortschr. Phys. 55, 491 (2007).

[13] M. Bianchi and E. Kiritsis, arXiv:hep-th/0702015.

[14] M. B. Green and M. Gutperle, J. High Energy Phys. 02 (2000) 014.

[15] M. Billo et al., J. High Energy Phys. 02 (2003) 045.

[16] C. Beasley and E. Witten, J. High Energy Phys. 02 (2006) 060.

[17] R. Blumenhagen, M. Cvetič, F. Marchesano, and G. Shiu, J. High Energy Phys. 03 (2005) 050.

[18] R. Blumenhagen and E. Plauschinn, J. High Energy Phys. 08 (2006) 031.

[19] G. Pradisi, arXiv:hep-th/0210088.

[20] E. Dudas and C. Timirgaziu, Nucl. Phys. B716, 65 (2005).
[21] L. Ibáñez and A. Uranga (private communication).

[22] M. Cvetič and R. Richter, Nucl. Phys. B762, 112 (2007).

[23] C. Beasley and E. Witten, J. High Energy Phys. 10 (2003) 065.

[24] E. I. Buchbinder, Phys. Lett. B 645, 281 (2007).

[25] D. Lüst and S. Stieberger, Fortschr. Phys. 55, 427 (2007).

[26] E. Witten, J. High Energy Phys. 02 (2000) 030.

[27] M.A. Shifman and A.I. Vainshtein, arXiv:hep-th/ 9902018.

[28] M. Cvetič and I. Papadimitriou, Phys. Rev. D 68, 046001 (2003).

[29] D. Lüst, P. Mayr, R. Richter, and S. Stieberger, Nucl. Phys. B696, 205 (2004).

[30] D. Cremades, L. E. Ibáñez, and F. Marchesano, J. High Energy Phys. 07 (2003) 038.

[31] M. Cvetič, I. Papadimitriou, and G. Shiu, Nucl. Phys. B659, 193 (2003).

[32] C.-M. Chen, V.E. Mayes, and D. V. Nanopoulos, Phys. Lett. B 648, 301 (2007).

[33] M. Cvetič, G. Shiu, and A. M. Uranga, Phys. Rev. Lett. 87, 201801 (2001).

[34] M. Cvetič, G. Shiu, and A. M. Uranga, Nucl. Phys. B615, 3 (2001).

[35] M. Cvetič, P. Langacker, and G. Shiu, Nucl. Phys. B642, 139 (2002).

[36] I. R. Klebanov and E. Witten, Nucl. Phys. B664, 3 (2003). 